\title{
Václav Fabian
}

Structural unambiguity of formal languages

Czechoslovak Mathematical Journal, Vol. 14 (1964), No. 3, 394-430

Persistent URL: http://dml.cz/dmlcz/100629

\section{Terms of use:}

(C) Institute of Mathematics AS CR, 1964

Institute of Mathematics of the Czech Academy of Sciences provides access to digitized documents strictly for personal use. Each copy of any part of this document must contain these Terms of use.

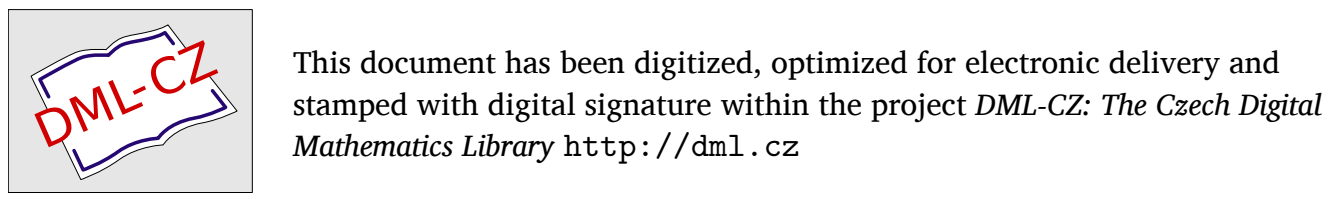




\title{
STRUCTURAL UNAMBIGUITY OF FORMAL LANGUAGES
}

\author{
VÁCLAV FABIAN, Praha
}

(Received April 12, 1963)

\section{INTRODUCTION AND SUMMARY}

The formal languages here considered form a class $\mathscr{C}$. which is contained in the class of all semi-Thue systems (see M. DAvis [7]; the restriction consists in that substitutions of other texts are possible only for texts of length 1 (i.e. for single symbols)) and contains the class of type 2 grammars of N. CHOMsKy [4] (context-free grammars). Especially ALGOL 60 (if considered without the limitations given in the non-formal parts of [1]) belongs to $\mathscr{C}$.

Both in definitions and proofs the concept of a (finite) sequence will be frequently used. If $\alpha$ is a sequence then $\lambda \alpha$ denotes its length and $\alpha i$ denotes its $i$-th element. By $\left[a_{1}, a_{2}, \ldots, a_{n}\right]$ we denote a sequence $\alpha$ of length $n$ such that $\alpha i=a_{i}$ for $i=1, \ldots, n$. Since elements of sequences may themselves be sequences, it is necessary to distinguish between an element $a$ and the sequence $[a]$ of length 1 .

To a given language $\mathscr{L}$ there will be associated the sets $\mathbf{a} \mathscr{L}, \mathbf{d} \mathscr{L}, \mathbf{a}_{\mathbf{t}} \mathscr{L}$ of its symbols, meta-symbols, terminal symbols, respectively, and a relation $\rightarrow$ between strings in $\mathscr{L}$ i.e. between finite sequences of symbols. (Here symbol means simply an element in the set $\mathbf{a} \mathscr{L}$. By a symbol we shall never - except in this sentence - mean any of the symbols which were used for printing this paper.) The set $\mathbf{g} \mathscr{L}$ of all grammatical elements of $\mathscr{L}$ is defined as the set of all $[A, t]$ such that $A \in \mathbf{d} \mathscr{L}$ and $A \rightarrow t$. If $[A, t]$ is a grammatical element of $\mathscr{L}$ we say that $t$ is a text (more explicitely, an $A$-text); a text is terminal if all of its symbols are terminal. A grammatical element $[A, t]$ is called terminal if $t$ is terminal. The relation $\rightarrow$ is derived from another simpler one, $\Rightarrow$, in the following way: $t_{1} \rightarrow t_{2}$ if $t_{2}$ can be obtained by replacing in $t_{1}$ a meta-symbol $A$ by a text $q$ and if $[A] \Rightarrow q$; or if $t_{2}$ can be obtained from $t_{1}$ by a finite number of successive substitutions of that kind. (Our $\rightarrow$ corresponds to Chomsky's $[4] \Rightarrow$.)

The intended application is that there will be a distinguished meta-symbol, say $s$, such that $s$-texts will be called self-contained texts or simply sentences. The remaining texts may be regarded as without meaning outside of grammatical elements. (For example in ALGOL 60, $s=\langle$ program〉 and the text $t$,,if $a$ then $b$ else $c$ " may have, 
roughly speaking, two meanings, if considered in the grammatical elements [ sion $\rangle, t],[\langle$ statement $\rangle, t]$. Similarly for $[\langle$ number $\rangle, 25],[\langle$ label $\rangle, 25]$.) Suppose $F$ ascribes some values to sentences, and also to non-self-contained texts if, in this latter case, the meta-symbol they are derived from is indicated. Then it is natural to formalize $F$ as a transformation defined on $\mathbf{g} \mathscr{L}$. The value ascribed to a sentence $t$ is then $F[s, t]$.

A concept of structure is defined in the following way: $[\alpha, \tau]$ is a structure of $[A, t]$ if either (i) $[A] \Rightarrow t$ and $\alpha=[A], \tau=[t]$, or if (ii) $[A] \Rightarrow \alpha \rightarrow t, \tau$ is a sequence of the same length $m$ as $\alpha$ and $[\alpha i] \rightrightarrows \tau i$ for $i=1, \ldots, m$ and

$$
t=\tau 1 \times \tau 2 \times \ldots \times \tau m,
$$

where $\times$ denotes juxtaposition of texts.

The concept of structure and that of structural description (N. CHOMSKY and M.P. SCHÜTZENBERGER [6]) are closely related (see Remark 5.8). In particular, every (terminal) grammatical element has exactly one structure (i.e. is structurally unambiguous) when and only when every (terminal) grammatical element has exactly one structural description; in this case we say that the language is (weakly) structurally unambiguous. The basic properties of structures are studied in Sec. 6. Theorem 6.7 gives what might be called a method of structural induction, frequently used later in proofs. Theorem 6.9 exhibits further equivalent definitions of the sets of all grammatical elements and texts. For a relation with a result of Chomsky and Schützenberger [6], see Remark 6.10.

Primarily, a semantics $S$ for a language is simply a transformation whose domain $\mathrm{d} S$ contains all sentences. (The range of $S$ may be various in different applications. It may be the set of texts of another language, the set of all real numbers and so on.) In Sec. 7 we shall introduce a more special concept of semantics. We shall require, first, that a semantics $S$ be defined on the set $\mathbf{g}_{\mathrm{t}} \mathscr{L}$ of all terminal grammatical elements (again, the value $S[s, t]$ is to be ascribed to every sentence $t$ ), and second, that the following condition hold: The value $S[A, t]$ depends, for every structure $[\alpha, \tau]$ of $[A, t]$, only on $A, \alpha$ and $\beta=\left[S_{0}[\alpha 1, \tau 1], \ldots, S_{0}[\alpha m, \tau m]\right]$, where $S_{0}$ is defined as follows: $S_{0}[\alpha j$, $\tau j]=\alpha j$ if $[\alpha j]=\tau j$ and $S_{0}[\alpha j, \tau j]=S[\alpha j, \tau j]$ otherwise. Roughly speaking, $S[A, t]$ is determined by the way in which $[A, t]$ is assembled from simpler grammatical elements, and by their semantical values. The rule which specifies this determination is called semantical. The main result of Sec. 7 is expressed in Theorem 7.5: A language $\mathscr{L}$ is weakly structurally unambiguous if and only if every semantical rule induces a semantics on $\mathscr{L}$. Another theorem of Sec. 7 gives conditions under which structural unambiguity and weak structural unambiguity are equivalent.

Sec. 8 contains some simple results concerning relations between languages.

The rest of the paper is devoted entirely to the study of conditions under which a given language is structurally unambiguous. It would be very desirable if there could be given an algorithm for deciding, for any language, whether or not it is (weakly) structurally unambiguous. Such an algorithm does not exist even for classes of lan- 
guages much more restricted than $\mathscr{C}$, see D. G. CANTOR [3], N. ChomsKy and M. P. Schützenberger [6] and A. Greibach [9]. Some sufficient conditions for structural unambiguity have already been given for less general languages (and not directly applicable e.g. to ALGOL 60) by H. Bottenbruch [2], F. Genuys [8] and N. ChomSKY and M.P. SCHÜTZENBERGER [6], the former two papers containing no proofs.

In Sec. 9 the concept of a reducing transformation is introduced as a transformation $\varrho$ defined on $\mathbf{g} \mathscr{L}$ and satisfying the conditions given in Definition 9.1. Roughly speaking $\varrho$ transforms a grammatical element $g=[A, t]$ into a $[A, V g]$ such that: $[A] \rightrightarrows$ $\rightrightarrows V g \nsupseteq t$ and that every structure of $g$ may be obtained from a structure of $[A, V g]$ if $[A] \neq V g$. Then $\varrho$ has the following properties: If $g$ is a grammatical element then $g$ is structurally unambiguous if either $\varrho g \notin \mathbf{g} \mathscr{L}$ or if $\varrho g$ is structurally unambiguous (Lemma 9.2), and $\mathscr{L}$ is structurally unambiguous if and only if each of its $\varrho$-invariant grammatical elements is structurally unambiguous (Theorem 9.3). Moreover, $\mathscr{L}$ is structurally unambiguous if and only if there is a reducing transformation $\varrho$ such that no grammatical element is $\varrho$-invariant (Theorem 9.5).

It may be difficult to construct a sufficiently powerful reducing transformation for which $\varrho g \neq g$ for all $g$. In such a case it is at least possible to simplify the original problem. Indeed Theorem 9.11 shows that, instead of $\mathscr{L}$, we may consider another language $\mathscr{L}_{0}$, which differs from $\mathscr{L}$ in that some meta-symbols occuring in texts $\alpha \in\{\alpha ;[A] \Rightarrow \alpha, A \in \mathbf{d} \mathscr{L}\}$ are replaced by new terminal symbols; roughly speaking in $\mathscr{L}_{0}$ it is not always possible to make substitutions of texts for meta-symbols of $\mathscr{L}$. Theorems 9.12 and 9.13 show conditions under which the structural unambiguity of a subset $\mathscr{L}_{1}$ of $\mathscr{L}$ is necessary and sufficient for that of $\mathscr{L}$.

In all these results the existence of a reducing transformation $\varrho$ with some additional properties plays an important role. Conditions which ensure this existence are studied in Theorem 9.6, Lemma 9.4 and in the whole Sec. 10. The results are applied in Sec. 11 to show that some meta-symbols may be deleted from a language without affecting the property of structural unambiguity. For example meta-symbols for which only texts in parantheses can be substituted have this property. Applications are also given in Examples 10.9 and 11.4. Other examples (usually given at the end of a section) are not intended to exhibit the usefulness of the obtained results but only to facilitate the reading of the paper.

The results of this paper were applied by Mr. J. GRUSKA to prove that ALGOL 60, after slight modifications of its syntax, is structurally unambiguous. This result is now being prepared for publication.

The author regrets that some of conditions investigated and some of the proofs are not very simple, but in several attempts he has made to simplify the paper he was unable to reach simplification without an essential decrease of the generality necessary to the intended practical applications. Moreover it was necessary to include a preparatory Sec. 4 concerning some operations on finite sequences and some elementary properties of these operations. Some changes in conventional notation seemed to be appropriate, too, for avoiding possible ambiguities. 
The importance of finding sufficient conditions for what, after formalizing, is very near or identical with our structural unambiguity, has been pointed out by H. BOTTENBRUCh [2], N. Chomsky [4], [5], R. Genuys[8], N. Chomsky and M. P. SchützenBERGER [6] and is very distinct in connection with automatic programming. For example many of the ambiguities of the semantics of ALGOL 60 (see Algol Bulletin from No. 10 on) were or are consequences of its structural ambiguities. It would be very important to have means for a rigorous definition of semantics for artificial languages such as ALGOL 60 (in this latter the precision of the description of the semantics is deeply below the rigour of the definition of syntax) or to have an algorithm for making compilers from such languages (see P. Z. INGERMAN [10]). Everywhere here structural unambiguity of the languages is very important (see also Theorem 7.5).

For a given language it may be quite obvious, without any formal study, that it is structurally unambiguous although this may be difficult to prove mathematically. This could be a good reason against the present study; only it may happen that a language the structural unambiguity of which is quite obvious is not, in fact, structurally unambiguous. It seems, for example, that the opinion that ALGOL 60 was structurally unambiguous was generally accepted when the Report [1] was published and also every time after a change was made to remove an ambiguity pointed out by an example.

\section{BASIC NOTATIONS}

3.1. Remark. In subsequent sections we shall deal with transformations and, especially, with finite sequences. Here we recall the basic concepts and introduce notations which will be useful later.

3.2. Sets. $\left\{a_{1}, a_{2}, \ldots, a_{n}\right\}$ denotes the set consisting of the indicated elements $a_{i} \cdot\{x ; \mathscr{C}(x)\}$ denotes the set of all such $x$ which satisfy condition $\mathscr{C}(x) .\{x ; \mathscr{C}(x, \Theta)\}$ is an abbreviation for $\{x$; there is a $\Theta$ such that $\mathscr{C}(x, \Theta)$ holds $\}$. $\Lambda$ denotes the empty set, $I_{0}$ and $I$ denote the set of all non-negative and positive integers, respectively, $I(i, j)=\left\{s ; s \in I_{0}, i \leqq s \leqq j\right\}, I(i)=I(1, i)$ for $i, j$ in $I_{0}$.

3.3. Pairs. By a pair $\left\langle x_{1}, x_{2}\right\rangle$ we mean the set $\left\{\left\{x_{1}\right\},\left\{x_{1}, x_{2}\right\}\right\}$. We see that $\left\langle x_{1}, x_{2}\right\rangle=\left\langle y_{1}, y_{2}\right\rangle$ if and only if $x_{1}=y_{1}, x_{2}=y_{2}$. (We use the concept of the pair in the definition of transformation, the concept of the transformation in the definition of the sequence. Hence we cannot say that a pair is a sequence of length two.)

3.4. Transformations. A transformation is a set $F$ (possibly empty) of pairs such that if $\left\langle x, y_{1}\right\rangle \in F,\left\langle x, y_{2}\right\rangle \in F$ then $y_{1}=y_{2}$. The domain $\mathbf{d} F$ and the range $\mathbf{r} F$ are defined by the relations

$$
\mathbf{d} F=\{x ;\langle x, y\rangle \in F\}, \quad \mathbf{r} F\{y ;\langle x, y\rangle \in F\} .
$$


The value $F x$ of $F$ at $x$ in $\mathbf{d} F$ is the unique element in $\mathbf{r} F$ such that $\langle x, F x\rangle \in F$. If $M \subset \mathbf{d} F$ then the symbol $F_{M}$ denotes the partial transformation $\{\langle x, F x\rangle ; x \in M\}$.

If $a, b$ are transformations, $c \in \mathbf{d} b$, it may be that $b c \in \mathbf{d} a$. In this case we write $a b c$ in the sense of $a(b c)$. On the other hand we do not delete parantheses in, e. g., $(a b) c$, which is the value of $a b$ at $c$, if $b \in \mathbf{d} a, a b$ is a transformation and $c \in \mathbf{d} a b$. Hence e.g. $a b(c d) e$ means $a(b((c d) e))$.

If $a, b$ are transformations, $\mathbf{d} a \supset \mathbf{r} b$, then $a \circ b$ is the compound transformation defined on $\mathbf{d} b$ by the relation $(a \circ b) x=a b x$.

\section{SEQUENCES, OPERATIONS WITH SEQUENCES AND DECOMPOSITIONS}

4.1. Remark. In this section some elementary concepts relating to finite sequences will be introduced. They will serve later for the study of texts, i.e., finite sequences of symbols.

4.2. Definition. $t$ is a sequence if $t$ is a transformation and $\mathbf{d} t=I(j)$ for some $j \in I_{0}$. The index $j$ is the length of $t$ and is denoted by $\lambda t$. We see that if $t$ is a sequence then $t$ is simply the set $\{\langle i, t i\rangle ; i \in I(\lambda t)\}$; it is convenient, however, to denote it also by $[t 1, t 2, \ldots, t \lambda t]$. Hence, conversely $\left[a_{1}, a_{2}, \ldots, a_{n}\right]$ denotes the sequence $\left\{\left\langle 1, a_{1}\right\rangle\right.$, $\left.\left\langle 2, a_{2}\right\rangle, \ldots,\left\langle n, a_{n}\right\rangle\right\}$. In view of the intended application, the values of a sequence will be also called symbols. If $M$ is a set of sequences, we define the sets

$$
\begin{aligned}
& \text { symb }_{\mathrm{b}} M=\{t 1 ; \lambda t \geqq 1, t \in M\}, \\
& \text { symb }_{\mathrm{e}} M=\{t \lambda t ; \lambda t \leqq 1, t \in M\}, \\
& \text { symb } M=\{t i ; t \in M, i \in \mathbf{d} t\}
\end{aligned}
$$

of all first symbols, last symbols, and symbols, respectively, of sequences in $M$.

If $M$ is a set and $n$ is a non-negative integer then we denote by $\mathbf{s}_{n} M$ the set of all sequences with symbols in $M$ and with length $n$, i.e.

$$
\mathbf{s}_{n} M=\{t ; t \text { is a sequence, } \lambda t=n, \operatorname{symb}\{t\} \subset M\} .
$$

Further we define

$$
\mathbf{s} M=\bigcup\left\{\mathbf{s}_{n} M ; n \geqq 0\right\}=\{t ; t \text { is a sequence, symb }\{t\} \subset M\} .
$$

Finally, if $t$ a sequence, $i, j$ are integers then we denote by $t^{(i, j)}$ the part (the $(i, j)$ -part) of $t$ defined by the relation

$$
t^{(i, j)}=\{\langle h+1-i, t h\rangle ; i \leqq h \leqq j\} .
$$

We have

$$
t^{(i, j)} h=t(i+h-1) \text { for every } h \in \mathbf{d} t^{(i, j)} .
$$


4.3. Definition. The product (also called the concacenation or juxtaposition) $t_{1} \times t_{2}$ of two sequences $t_{1}$ and $t_{2}$ is defined by

$$
t_{1} \times t_{2}=t_{1} \cup\left\{\left\langle i+\lambda t_{1}, t_{2} i\right\rangle ; i \in \mathbf{d} t_{2}\right\} .
$$

For a set $M, \mathbf{s} M$ with the operation $\times$ is an associative non-commutative semi-group with both-sided cancellation and with unit element $\Lambda$, i.e. we have for every $a, b, c$ in $\mathbf{s} M$ :

$$
(a \times b) \times c=a \times(b \times c)
$$

$$
\text { if } a \times b=a \times c \text { or } b \times a=c \times a \text { then } b=c \text {, }
$$

$$
a \times b=a \text { or } b \times a=a \text { if and only if } b=\Lambda \text {. }
$$

We also write $a \times b \times c$ for $(a \times b) \times c$. If $\tau \in \mathbf{s s} M$ then we write $\prod \tau$ or $\prod_{i=1}^{\lambda \tau} \tau i$ for $\tau 1 \times \tau 2 \times \ldots \times \tau \lambda \tau$.

4.4. Definition. $\tau$ is a decomposition if $\tau \in \mathbf{s s} M$ for some set $M$, i. e. if $\tau$ is a sequence of sequences. We say that $\tau$ is a decomposition of $t$ if $\prod \tau=t$. Next, $x$ is an index-sequence if it is a sequence, if $\mathbf{r} x \subset I, x 1=1$ and if $x$ is, as a function, nondecreasing. $x$ is an index-decomposition of a sequence $t$ if $x$ is an index-sequence and if

$$
x \lambda x=\lambda t+1 .
$$

If $\tau$ is a decomposition we define the index-decomposition $\mathbf{\tau}$ of $\prod \tau$ by $\iota \tau=x$, where

$$
\lambda x=\lambda \tau+1
$$

and

$$
x j=1+\lambda \prod \tau^{(1, j-1)} \text { for } j \in \mathbf{d} x .
$$

Note that

$$
\mathbf{r} x \subset I\left(\lambda \prod \tau+1\right) .
$$

On the other hand, if $x$ is an index-decomposition of a sequence $t$ then we denote by $\boldsymbol{\delta}(t, x)$ that decomposition $\tau$ of $t$ for which

$$
\tau j=t^{(x j, x(j+1)-1)} \text { for } j \in \mathbf{d} \tau .
$$

A decomposition $\tau$ is said to be primitive if $\lambda \tau=\lambda \prod \tau$ and $\tau i=\left[\left(\prod \tau\right) i\right]$ for every $i \in \mathbf{d} \tau$. The unique primitive decomposition of a sequence $t$ will be denoted by $\boldsymbol{\delta}_{p} t$.

4.5. Lemma. Let $\tau$ be a decomposition of $t$. Then the relations $x=\imath \tau$ and $\tau=$ $=\delta(t, x)$ are equivalent mutually and also to

$$
\prod \tau^{(i, j)}=t^{(x i, x(j+1)-1)} \text { for } i, j \in \mathbf{d} \tau .
$$


Proof. Suppose $x=\mathbf{\imath} \tau$. From (4.4.3) we get (1) if $i=1$; otherwise, if $i>1$, then $\prod \tau^{(1, i-1)}=t^{(1, x i-1)}, \prod \tau^{(1, j)}=t^{(1, x(j+1)-1}$ and by left cancellation we get (1). If $i=j$ then (1) reduces to (4.4.5) since $\prod \tau^{(j, j)}=\tau j$. From (4.4.5) we obtain (4.4.3) easily. Hence $x=\imath \tau$ implies (1), (1) implies $\tau=\delta(t, x)$ and this last equality implies $x=\mathbf{\imath} \tau$.

4.6. Definition. Let $\tau_{1}, \tau_{2}$ be two decompositions, $\lambda \prod \tau_{1}=\lambda \tau_{2}$. Then we define a new decomposition $\tau_{1} \otimes \tau_{2}$ as the decomposition of $\prod \tau_{2}$ with the index-decomposition

$$
\mathbf{t}\left(\tau_{1} \otimes \tau_{2}\right)=\left(\mathbf{\imath} \tau_{2}\right) \circ\left(\mathbf{\iota} \tau_{1}\right)
$$

(We remark that the right-hand side of (1) has a meaning because by (4.4.4) $\left.\mathbf{r} \mathbf{\iota} \tau_{1} \subset I\left(\lambda \prod \tau_{1}+1\right)=I\left(\lambda \tau_{2}+1\right)=\mathbf{d} \mathbf{\imath} \tau_{2}.\right)$

4.7. Lemma. Let $\tau_{1}, \tau_{2}, \tau$ be decompositions, $\tau=\tau_{1} \otimes \tau_{2}, x_{1}=\mathbf{\imath} \tau_{1}$. Then

$$
\mathbf{d} \tau=\mathbf{d} \tau_{1}
$$

and

$$
\tau i=\prod \tau_{2}^{\left(x_{1} i, x_{1}(i+1)-1\right)} \text { for every } i \in \mathbf{d} \tau .
$$

Proof. According to (4.4.2) and (4.6.1) we have $\mathbf{d} \tau=I(\lambda \mathbf{\mathbf { l }} \tau-1)=I\left(\lambda x_{1}-1\right)=$ $=\mathbf{d} \tau_{1}$ and (1) holds. If we denote $x_{2}=\mathbf{\imath} \tau_{2}$ then by definition of $\tau_{1} \otimes \tau_{2}$ we obtain that $\tau i=\left(\prod \tau_{2}\right)^{\left(x_{2} x_{1} i, x_{2} x_{1}(i+1)-1\right)}$. This equals the right-hand side of (4.5.1) with $x_{2}$, $\prod \tau_{2}, \tau_{2}, x_{1} i, x_{1}(i+1)-1$ substituted for $x, t, \tau, i, j$. Since these substitutions change the left-hand side of (4.5.1) to the right-hand side of (2), this last equality is proved.

4.8. Lemma. Let $\tau_{1}$ be a decomposition of a decomposition $\tau_{2}$. Then $\tau_{1} \otimes \tau_{2}$ is defined and if $\tau=\tau_{1} \otimes \tau_{2}$ then

$$
\tau h=\prod \tau_{1} h \text { for every } h \in \mathbf{d} \tau .
$$

Proof. Because $\prod \tau_{1}=\tau_{2}$, we have $\lambda \prod \tau_{1}=\lambda \tau_{2} ; \tau$ is defined and (1) is an immediate consequence of (4.7.2) and (4.4.5).

4.9. Definition. Let $\tau, \tau_{2}$ be two decompositions. We say that $\tau_{2}$ is finer than $\tau$ if there is a decomposition $\tau_{1}$ such that $\tau=\tau_{1} \otimes \tau_{2}$. (We may require that $\tau_{1}$ be a decomposition of $\tau_{2}$ since $\tau_{1}^{\prime}=\delta\left(\tau_{2}, \iota \tau_{1}\right)$ has this property and $\tau=\tau_{1}^{\prime} \otimes \tau_{2}$.)

4.10. Lemma. Let $\tau, \tau_{2}$ be two decompositions, $x=\mathbf{\imath} \tau, x_{2}=\mathbf{\imath} \tau_{2}$. Then $\tau_{2}$ is finer than $\tau$ if and only if

$$
\prod \tau_{2}=\prod \tau, \quad \mathbf{r} x \subset \mathbf{r} x_{2} .
$$

Proof. If $\tau_{2}$ is finer than $\tau$ then there are $\tau_{1}, x_{1}=\imath \tau_{1}$ such that $\tau=\tau_{1} \otimes \tau_{2}$, which implies $\prod \tau=\prod \tau_{2}, x=x_{2} \circ x_{1}, \mathbf{r} x \subset \mathbf{r} x_{2}$ and (1) holds.

On the other hand let (1) hold and define $x_{1}$ on $\mathbf{d} x$ by the relations $x_{1} 1=1$, 
$x_{1} h=\operatorname{Max}\left\{i ; i \in \mathbf{d} x_{2}, x_{2} i=x h\right\}$ for $h \neq 1$. Then $x_{1} 1=1, \quad x_{1} \lambda x_{1}=x_{1} \lambda x=$ $=\operatorname{Max}\left\{i ; i \in \mathbf{d} x_{2}, x_{2} i=x \lambda x\right\}=\lambda x_{2}=\lambda \tau_{2}+1$, since $x \lambda x=\lambda \prod \tau+1=\lambda \prod \tau_{2}+$ $+1=x_{2} \lambda x_{2}$. Moreover, $x_{1}$ is non-decreasing and hence it is an index-decomposition of $\tau_{2}$. We have $x=x_{2} \circ x_{1}$, hence $\tau=\delta\left(\tau_{2}, x_{1}\right) \otimes \tau_{2}$ and $\tau_{2}$ is finer than $\tau$.

4.11. Lemma. Let $\tau_{1}, \tau_{2}$ be two decompositions of a decomposition $\xi$, let $\Lambda \notin$ $\notin$ symb $\{\xi\}$ and

$$
\tau_{1} \otimes \xi=\tau_{2} \otimes \xi
$$

Then $\tau_{1}=\tau_{2}$.

Proof. Put $y=\mathbf{\iota} \xi, x_{i}=\mathbf{\imath} \tau_{i}$. From (1) and (4.6.1) we get $y \circ x_{1}=y \circ x_{2}$ and, since $y$ is increasing, $x_{1}=x_{2}$ and $\tau_{1}=\delta\left(\xi, x_{1}\right)=\delta\left(\xi, x_{2}\right)=\tau_{2}$.

4.12. Examples. Let $M$ be a set, $a, b, c \in M$. Then $t=[a, b, c] \in \mathbf{s}_{3} M, t^{(2,3)}=$ $=[b, c], t \times[a]=[a, b, c, a]$ and $[[a],[b, c]], \tau=[[a, b],[c], \Lambda]$ and $[[a]$, $[b],[c]]$ are three examples of decompositions of $t$; the third is the primitive decomposition $\boldsymbol{\delta}_{p} t$. If $x=[1,3,4,4]$ then $x$ is an index-decomposition of every sequence of length 3. We have $x=\imath t, \tau=\delta(t, x)$ and also $\delta[\tau, x]=[[[a, b],[c]],[\Lambda], \Lambda]$.

If $\xi=[[1],[2,3]]$ then $\lambda \prod \xi=\lambda[1,2,3]=3=\lambda \tau$ and $\xi \otimes \tau$ is defined. Since $\tau_{1} \otimes \tau=\xi \otimes \tau$ if $\mathbf{\imath} \tau_{1}=\mathbf{\imath} \xi$, we may put $\tau_{1}=\boldsymbol{\delta}(\tau,[1,2,4])=[[[a, b]],[[c], \Lambda]]$ and by (4.8.1),

$$
\begin{aligned}
& \left(\tau_{1} \otimes \tau\right) 1=\prod \tau_{1} 1=\prod[[a, b]]=[a, b], \\
& \left(\tau_{1} \otimes \tau\right) 2=\prod \tau_{1} 2=\prod[[c], \Lambda]=[c]
\end{aligned}
$$

and $\xi \otimes \tau=[[a, b],[c]]$.

By Definition 4.9, $[[a, b],[c], \Lambda]$ is finer than $[[a, b],[c]]$. Similarly $[[a, b],[c]]$ is finer than $[[a, b, c]]$.

\section{LANGUAGE}

5.1. Remark. Before giving a formal definition the following remarks may be convenient. First what we call here a language may be also called a grammar of a language. Secondly only terminal texts are texts in the proper sense; texts which are not terminal are only suitable means for expressing the syntactical rules. Thirdly a primary concept is that of the relation $\Rightarrow$ which is here expressed in terms of a transformation which may be identified with the language itself. Finally we note that it is not necessary for our study to distinguish between sentences and other (non-selfcontained) texts.

5.2. Definition. $\mathscr{L}$ is a language if $\mathscr{L}$ is a transformation, if there exists a set $\mathrm{A}$ such that

$$
\mathbf{d} \mathscr{L} \subset \mathbf{A}, \quad \mathbf{r} \mathscr{L} \subset\{M ; A \neq M \subset \mathbf{s A}\}
$$


and if

$[A] \notin \mathscr{L} A$ for every $A \in \mathbf{d} \mathscr{L}$.

With a language $\mathscr{L}$ we associate the alphabet $\mathbf{a} \mathscr{L}$ defined as the smallest set $\boldsymbol{A}$ for which (1) holds. The elements of a $\mathscr{L}$ will be called symbols of $\mathscr{L}$. The elements of the sets $\mathbf{d} \mathscr{L}, \mathbf{a}_{\mathrm{t}} \mathscr{L}=\mathbf{a} \mathscr{L}-\mathbf{d} \mathscr{L}, \boldsymbol{\sigma} \mathscr{L}=\mathbf{s a} \mathscr{L}, \boldsymbol{\sigma}_{\mathrm{t}} \mathscr{L}=\mathbf{s} \mathbf{t}_{\mathrm{t}} \mathscr{L}$ are called meta-symbols, terminal symbols, strings, terminal strings, respectively, of $\mathscr{L}$.

We shall write $\mathscr{L}:[A] \Rightarrow b$ as an abbreviation for $A \in \mathbf{d} \mathscr{L}, b \in \mathscr{L} A$. We write $\mathscr{L}: a \equiv b$ if either $\mathscr{L}: a \Rightarrow b$ or $a=b$. We write $\mathscr{L}: a \rightarrow b$ if there are strings $q_{1}, q_{2}, q_{3}$ and a metasymbol $A$ such that $a=q_{1} \times[A] \times q_{3}, b=q_{1} \times q_{2} \times q_{3}$, $q_{2} \in \mathscr{L} A$. (I. e. if $b$ may be obtained by substituting $q_{2}$ for the $\left(\lambda q_{1}+1\right)$-th symbol (which is $A$ ) of $a$.) $\mathscr{L}: a \Longrightarrow b$ means that either $a=b$ or $\mathscr{L}: a \rightarrow b$.

We say that $\sigma$ is a pseudoderivation (in $\mathscr{L}$ of $\sigma \lambda \sigma$ from $\sigma 1$ ) if $\sigma$ is a sequence, $\lambda \sigma \geqq 1$, $\mathscr{L}: \sigma i \rightarrow \sigma(i+1)$ for every $i \in I(\lambda \sigma-1)$. A pseudoderivation is said to be a derivation if its length is greater than 1 . We write $\mathscr{L}: a \rightarrow b$ if there is a derivation in $\mathscr{L}$ of $b$ from $a$ and we write $\mathscr{L}: a \Longrightarrow b$ if $a \rightarrow b$ or $a=b$.

If there is no danger of misunderstanding, the symbol specifying the language will be deleted in $\Rightarrow$, $\rightarrow$ etc.

Now we define the sets

$$
\begin{aligned}
& \mathbf{t}(\mathscr{L}, A)=\{t ;[A] \rightarrow t\}, \\
& \mathbf{t}_{\mathbf{t}}(\mathscr{L}, A)=\mathbf{t}(\mathscr{L}, A) \cap \boldsymbol{\sigma}_{\mathrm{t}} \mathscr{L}, \\
& \mathbf{t} \mathscr{L}=\bigcup\{\mathbf{t}(\mathscr{L}, A) ; A \in \mathbf{d} \mathscr{L}\}, \\
& \mathbf{t}_{\mathbf{t}} \mathscr{L}=\mathbf{t} \mathscr{L} \cap \sigma_{\mathrm{t}} \mathscr{L}
\end{aligned}
$$

of all $A$-texts, terminal $A$-texts, texts, terminal texts, respectively, of $\mathscr{L}$. We have, of course, $\mathbf{t}(\mathscr{L}, A)=\Lambda$ for $A$ not in $\mathbf{d} \mathscr{L}$.

5.3. Remark. The concept of language just introduced is a generalization of the concept of a type 2 grammar of N. CHOMSKY [4].

First finiteness of $\mathbf{d} \mathscr{L} \cup \cup \mathbf{r} \mathscr{L}$ seems to be irrelevant to this study and, therefore, is not assumed. Secondly Restriction 2 in [4] excludes $[A] \Rightarrow \Lambda$; in [6] N. Chomsky and M. P. Schützenberger assume additionally that $[A] \Rightarrow[B]$ for no metasymbols $A, B$. Both suppositions are violated by ALGOL 60 and not made here. As stated in [6], the suppositions do not affect the generative capacity of the language; this capacity, however, is not the object of our study.

5.4. Convention. In the sequel it will be supposed that a language $\mathscr{L}$ is given. Later we shall, however, consider more than one language. Then the concepts relating to a language must be specified by indicating the language they refer to.

5.5. Lemma. The relations $\rightarrow$ and $\rightrightarrows$ are multiplicative in the sense that if $\tau_{1}$ and $\tau_{2}$ are two decompositions such that $\tau_{1} i \rightrightarrows \tau_{2}$ i for every $i \in \mathbf{d} \tau_{1}=\mathbf{d} \tau_{2}$ then $\prod \tau_{1} \rightrightarrows$ $\rightrightarrows \prod \tau_{2} ;$ if, moreover, $\tau_{1} i \rightarrow \tau_{2} i$ for at least one $i$ then $\prod \tau_{1} \rightarrow \prod \tau_{2}$. 
Proof. Obvious.

5.6. Definition. The set of all grammatical elements of $\mathscr{L}$ is defined as the set

$$
\mathbf{g} \mathscr{L}=\{[A, t] ; A \in \mathbf{d} \mathscr{L}, t \in \mathbf{t}(\mathscr{L}, A)\} ;
$$

the set of all terminal grammatical elements is defined by

$$
\mathbf{g}_{\mathbf{t}} \mathscr{L}=\left\{[A, t] ; A \in \mathbf{d} \mathscr{L}, t \in \mathbf{t}_{\mathbf{t}}(\mathscr{L}, A)\right\} .
$$

A pseudoderivation (derivation) of $t_{1}$ from $t_{0}$ is called normal if there is no shorter pseudoderivation (derivation) of $t_{1}$ from $t_{0}$.

The depth $\delta$ will be defined as a transformation on $\mathbf{g} \mathscr{L}$ such that $\delta[A, t]$ is the

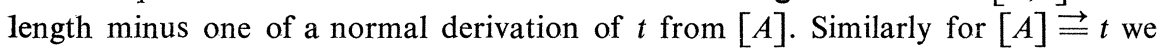
denote by $\delta_{0}[A, t]$ the length minus one of a normal pseudoderivation of $t$ from $[A]$.

5.7. Definition. Let $\tau$ be a decomposition, $\prod \tau \in \mathbf{t} \mathscr{L}$. We say that $\alpha$ is a naming for $\tau$ if $\alpha \in \sigma \mathscr{L}$ and if

$$
\lambda \alpha=\lambda \tau \text { and }[\alpha i] \rightrightarrows \tau i \text { for every } i \in \mathbf{d} \alpha .
$$

We say that $\tau$ is an $\alpha$-decomposition if $\alpha$ is a naming for $\tau$.

We say that $[\alpha, \tau]$ is a structure of a grammatical element $[A, t]$ if

$$
\tau \text { is an } \alpha \text {-decomposition of } t \text { and either }
$$

$$
[A]=\alpha,[A] \Rightarrow t,[t]=\tau
$$

or

$$
[A] \Rightarrow \alpha \rightarrow t \text {. }
$$

5.8. Remark. The concepts of structure and of structural description (Chomsky and Schützenberger [6]) are closely related. We shall show this only informally on the example from p. 122 in [6]. As in our other examples we shall denote a sequence $\left[a_{1}, a_{2}, \ldots, a_{n}\right]$ of symbols $a_{i}$ also by $a_{1} a_{2} \ldots a_{n}$. Since the symbols here are English words or abbrevations and are denoted by letter strings they will be separated by a space.

The relations (5), [6] may be rewritten, in our formulation, as

$$
S \Rightarrow N P V P ; N P \Rightarrow \text { they }
$$

and so on. Now when we delete, in a structural description, all labelled brackets except those which are of depth 1 and 2, we obtain essentially what we call a structure. Thus we get from (6), [6]

$$
[s[N P \text { they }][V P \text { are flying planes }]]
$$

and $[\alpha, \tau]$ with

$$
\alpha=N P V P, \quad \tau=[\text { they, are flying planes] }
$$

is a structure of the grammatical element [S, they are flying planes]. 
Conversely, from a structure $[\alpha, \tau]$ of a grammatical element $[A, t]$ and from structures of those $[\alpha i, \tau i]$ which are grammatical elements, and so on, we obtain a structural description.

For example the grammatical element

$$
[V P \text {, are flying planes] }
$$

has a structure $[\alpha, \tau]$ where

$$
\alpha=\operatorname{VerbNP}, \quad \tau=\text { [are flying, planes] }
$$

which may be written as

$$
\text { [VP } \left.[\text { Verb } \text { are flying }]\left[{ }_{N P} \text { planes }\right]\right] \text {. }
$$

This substituted for $\left[V_{P} \ldots\right]$ in (1), leads to the structural description (6), [6].

The grammatical element (2), however, also has another structure

$$
\text { [are } N P \text {, [are, flying planes]] }
$$

and $[N P$, flying planes $]$ has the structure

$$
\text { [Adj } N \text {, [flying, planes]] }
$$

and these structures lead to the structural description (7), [6].

5.9. Examples. Let $a, n$ be two different elements and

$$
\mathscr{L}=\{\langle a,\{n, a n\}\rangle,\langle n,\{0,1\}\rangle\} .
$$

Then $\mathbf{d} \mathscr{L}=\{a, n\}, \mathbf{r} \mathscr{L}=\{\{n, a n\},\{0,1\}\}, \mathbf{a} \mathscr{L}=\{a, n, 0,1\}, \mathbf{a}_{\mathrm{t}} \mathscr{L}=\{0,1\}$. Now $\sigma_{1}=[a, a], \quad \sigma_{2}=[a, a n, a n n, a n 0, a 10], \quad \sigma_{3}=[a, a n, a 0, a n 0, a 10]$ are three pseudoderivations, and $\sigma_{2}$ and $\sigma_{3}$ two derivations, both of $a 10$ from $a$. The $n$-texts are 0 and 1 . The terminal $a$-texts are non-empty sequences of zeros and ones. If $\alpha=a n$, $\tau=[a n, 0]$ then $\alpha$ is a naming for $\tau$ and $[\alpha, \tau]$ is a structure of $[a, a n 0]$. The unique structure of $[n, 1]$ is $[n,[1]]$. (A more precise notation, used outside of examples, would be $[n,[1]]$ and $[[n],[[1]]]$ in the last sentence.) It can be easily seen that $\mathscr{L}$ is structurally unambiguous.

5.10. Example. Let $\mathscr{L}=\{\langle a,\{0,1, a a\}\rangle\}$. Then this language with a single meta-symbol $a$ has the same set of terminal texts as the language from the preceding. example, but is structurally ambiguous.

\section{EXISTENCE AND PROPERTIES OF STRUCTURES}

6.1. Lemma. Let $\tau_{i}$ be a $t_{i-1}$-decomposition of $t_{i}$ for $i=1,2$. Then $\tau=\tau_{1} \otimes \tau_{2}$ is a $t_{0}$-decomposition of $t_{2}$.

Moreover, if $\zeta_{1}=\delta\left(\tau_{2}, \mathbf{\imath} \tau_{1}\right)$, then $\mathbf{d} \zeta_{1}=\mathbf{d} t_{0^{\prime}}=\mathbf{d} \tau_{1}$ and, for every $i \in \mathbf{d} \zeta_{1}, \zeta_{1} i$ is: a $\tau_{1}$ i-decomposition of $\tau i$. 
Proof. Put $x_{1}=\mathbf{\imath} \tau_{1}$. Then $\tau=\zeta_{1} \otimes \tau_{2}$ and $\zeta_{1} i=\tau_{2}^{\left(x_{1} i, x_{1}(i+1)-1\right)}$ is a decomposition of $\tau i$ because $\tau i=\prod \zeta_{1} i$ (see Lemma 4.7). Furthermore $\tau_{1} i=t^{\left(x_{1} i, x_{1}(i+1)-1\right)}$, hence $\lambda \tau_{1} i=\lambda \zeta_{1} i$ and, because by assumption $\left[t_{1} j\right] \rightrightarrows \tau_{2} j$ for every $j \in \mathbf{d} t_{1}$, we have $\left[\left(\tau_{1} i\right) j\right] \supseteqq\left(\zeta_{1} i\right) j$ for every $j \in \mathbf{d} \zeta_{1} i$. Hence $\xi_{1} i$ is a $\tau_{1} i$-decomposition of $\tau i$ and the second assertion of the lemma holds. By multiplicativity (see Lemma 5.5) it implies the first assertion.

6.2. Definition. A $t_{1}$-decomposition $\tau$ is called immediate if $\left[t_{1} i\right] \Rightarrow \tau i$ for every $i \in \mathbf{d} t_{1}$ and $\left[t_{1} i\right] \neq \tau i$ for at most one $i \in \mathbf{d} t_{1}$.

6.3. Lemma. Let $t_{1} \rightrightarrows t_{2}$, let $\sigma$ be a normal pseudoderivation of $t_{2}$ from $t_{1}$. Then for every $t_{1}$-decomposition $\tau$ of $t_{2}$ we have

$$
\lambda \sigma-1 \leqq \sum_{i=1}^{\lambda t_{1}} \delta_{0}\left[t_{1} i, \tau i\right]
$$

and there is a $t_{1}$-decomposition $\tau$ of $t_{2}$ such that

$$
\lambda \sigma-1=\sum_{i=1}^{\lambda t_{1}} \delta_{0}\left[t_{1} i, \tau i\right]
$$

Proof. The assertion is trivial if $t_{1}=t_{2}$. Hence we may assume $t_{1} \rightarrow t_{2}, t_{1} \neq t_{2}$. First prove the first assertion. Let $\sigma_{i}$ be a shortest pseudoderivation of $\tau i$ from $\left[t_{1} i\right]$ for $i=1,2, \ldots, \lambda t_{1}$, so that $\delta_{0}\left[t_{1} i, \tau i\right]=\lambda \sigma_{i}-1$. Put

$$
v_{i}=\prod_{j=1}^{\lambda \sigma_{i}}\left[t^{(1, i-1)} \times \sigma_{i} j \times \prod \tau^{\left.(i+1), \lambda t_{1}\right)}\right] .
$$

Then $v_{i}$ is a pseudoderivation of $t^{(1, i-1)} \times \prod \tau^{\left(i, \lambda t_{1}\right)}$ from $t^{(1, i)} \times \prod \tau^{\left(i+1, \lambda t_{1}\right)}$ and

$$
\sigma_{0}=\left[t_{1}\right] \times \prod_{i=1}^{\lambda t_{1}} v_{\lambda t_{1}+1-i}^{\left(2, \lambda v_{i}\right)}
$$

is a pseudoderivation of $t_{2}=\prod \tau$ from $t_{1}$ of length $1+\sum_{i=1}^{\lambda t_{1}}\left(\lambda \sigma_{i}-1\right)=1+$ $+\sum_{i=1}^{\lambda t_{1}} \delta_{0}\left[t_{1} i, \tau i\right]$. By assumption $\lambda \sigma \leqq \lambda \sigma_{0}$ which proves $(1)$.

Now we shall prove (2) for a suitably chosen $\tau$, by the induction on the length $\lambda \sigma$. If $\lambda \sigma=1$ then $t_{1}=t_{2}$ and (2) holds. Suppose (2) holds if $\lambda \sigma<k$, let $\lambda \sigma=k>1$. By the inductive assumption there is a $t_{1}$-decomposition $\tau_{1}$ of $u=\sigma(\lambda \sigma-1)$ for which

$$
\lambda \sigma-2=\sum_{i=1}^{\lambda t_{1}} \delta_{0}\left[t_{1} i, \tau_{1} i\right]
$$

and an $u$-decomposition $\tau_{2}$ of $t_{2}$ for which $[u j]=\tau_{2} j$ for all $j$ except one $j_{0}$, for which $\left[u j_{0}\right] \Rightarrow \tau_{2} j_{0}$. By Lemma 6.1, $\tau=\tau_{1} \otimes \tau_{2}$ is a $t_{1}$-decomposition of $t_{2}$. We have (see 
(4.7.2)) $\tau i=\prod \tau_{2}{ }^{\left(x_{1} i, x_{1}(i+1)-1\right)}$ for every $i \in \mathbf{d} t_{1}$ where $x_{1}=\mathbf{t} \tau_{1}$. For exactly one $i_{0}$ we have $x_{1} i_{0} \leqq j_{0}<x_{1}\left(i_{0}+1\right)$. Then

$$
\begin{gathered}
\tau i_{0}=\prod \tau_{2}{ }^{\left(x_{1} i_{0}, j_{0}-1\right)} \times \prod \tau_{2}^{\left(j_{0}, j_{0}\right)} \times \prod \tau_{2}^{\left(j_{0}+1, x_{1}\left(i_{0}+1\right)-1\right)}= \\
=u^{\left(x_{1} i_{0}, j_{0}-1\right)} \times \tau_{2} j_{0} \times u^{\left(j_{0}+1, x_{1}\left(i_{0}+1\right)-1\right)}
\end{gathered}
$$

and

$$
\left[t_{1} i_{0}\right] \supseteqq \tau_{1} i_{0}=u^{\left(x_{1} i_{0}, x_{1}\left(i_{0}+1\right)-1\right)} \rightarrow \tau i_{0} .
$$

Hence $\delta_{0}\left[t_{1} i_{0}, \tau i\right] \leqq \delta_{0}\left[t_{1} i_{0}, \tau_{1} i_{0}\right]+1$. For $i \neq i_{0}$ we have $\tau_{1} i=\tau i$. With (3) this yields

$$
\sum_{i=1}^{\lambda t_{1}} \delta_{0}\left[t_{1} i, \tau i\right] \leqq \lambda \sigma-1 ;
$$

since the opposite inequality (1) has already been proved, (2) holds and the proof is complete.

\subsection{Lemma.}

(1) If $[A] \rightarrow t_{1} \rightrightarrows t_{2}$ and $\tau$ is a $t_{1}$-decomposition of $t_{2}$ then

$$
\delta\left[A, t_{2}\right] \leqq \delta\left[A, t_{1}\right]+\sum_{i=1}^{\lambda t_{1}} \delta_{0}\left[t_{1} i, \tau i\right] .
$$

(2) If $\sigma$ is a normal derivation of $t_{2}$ from $[A]$ and $[A] \neq t_{1} \in \operatorname{symb}\{\sigma\}$,

then there is a $t_{1}$-decomposition $\tau$ such that

$$
\delta\left[A, t_{2}\right]=\delta\left[A, t_{1}\right]+\sum_{i=1}^{\lambda t_{1}} \delta_{0}\left[t_{1} i, \tau i\right] .
$$

Proof. Let $\sigma$ be as in Lemma 6.3. Then $\delta\left[A, t_{2}\right] \leqq \delta\left[A, t_{1}\right]+\lambda \sigma-1$ and (1a) follows from (6.3.1).

If (2) holds then $t_{1}=\sigma i$ for an $i \in I(2, \lambda \sigma), \sigma^{(1, i)}$ is a normal derivation of $t_{1}$ from $[A], \sigma^{(i, \lambda \sigma)}$ is a normal pseudoderivation of $t_{2}$ from $t_{1}, \delta\left[A, t_{2}\right]=\lambda \sigma-1, \delta\left[A, t_{1}\right]=$

$=i-1$ and by Lemma 6.3, there is a $t_{1}$-decomposition $\tau$ of $t_{2}$ such that $\sum_{i=1}^{\lambda t_{1}} \delta_{0}\left[t_{1} i\right.$, $\tau i]=\lambda \sigma-i$. For such a $\tau(2 \mathrm{a})$ holds.

6.5. Theorem. Let $g=[A, t] \in \mathbf{g} \mathscr{L}$. Then there is a structure $[\alpha, \tau]$ of $g$ such that either

$$
[A] \Rightarrow t, \quad[\alpha, \tau]=[[A],[t]]
$$

or

$$
\delta[A, t]=1+\sum_{i=1}^{\lambda \alpha} \delta_{0}[\alpha i, \tau i]
$$


Proof. If $A \Rightarrow t$ then by (5.7.3a) $[[A],[t]]$ is a structure satisfying (1). In the remaining case let $\sigma$ be a normal derivation of $t$ from $A$. We have $\lambda \sigma>2$. Put $\alpha=\sigma 2$ so that (5.7.3b) holds. By Lemma 6.4, there is an $\alpha$-decomposition $\tau$ of $t$ for which

$$
\delta[A, t]=\delta[A, \alpha]+\sum_{i=1}^{\lambda \alpha} \delta_{0}[\alpha i, \tau i]
$$

and (2) holds since $\delta[A, \alpha]=1$.

6.6. Definition. Let $M \subset \mathbf{g} \mathscr{L}$, let $[\alpha, \tau]$ be a structure of a grammatical element $[A, t]$. We say that $[\alpha, \tau]$ is weakly $M$-regular if for every $i \in \mathbf{d} \alpha$ we have either $[\alpha i]=$ $=\tau i$ or $[\alpha i, \tau i] \in M$. We say that $[\alpha, \tau]$ is $M$-regular if it is weakly $M$-regular and

$$
\delta[A, t]=1+\sum_{i=1}^{\lambda \alpha} \delta_{0}[\alpha i, \tau i] .
$$

6.7. Theorem. Let $M \subset \mathbf{g} \mathscr{L}$ satisfy the following two conditions:

$$
M \supset\{[A, t] ;[A] \Rightarrow t\} .
$$

(2) If a grammatical element $g$ has an M-regular structure then $g \in M$.

Then $M=\mathbf{g} \mathscr{L}$.

Proof. Let $[A, t] \in \mathbf{g} \mathscr{L}$. If $\delta[A, t]=1$ then $[A] \Rightarrow t$ and $[A, t] \in M$ according to (1). Suppose $[A, t] \in M$ whenever $\delta[A, t] \leqq k-1$, let $\delta[A, t]=k \geqq 2$. Then by Theorem 6.5 there is a structure $[\alpha, \tau]$ satisfying (6.5.2). By the inductive assumption, $[\alpha, \tau]$ is $M$-regular and by $(2),[A, t] \in M$. This completes the proof by induction of $\mathbf{g} \mathscr{L} \subset M$.

6.8. Remark. The preceding theorem will often be used in a weaker form with condition (6.7.2) replaced by

(1) If a grammatical element $g$ has a weakly $M$-regular structure then $g \in M$. Clearly (1) implies (6.7.2). Hence conditions (6.7.1) and (1) imply $g \in M$.

The next theorem shows other ways of constructing the sets $\mathbf{g} \mathscr{L}$ and $\mathbf{g}_{\mathrm{t}} \mathscr{L}$ than are those given by the definition. In particular, for constructing $\mathbf{g}_{\mathbf{t}} \mathscr{L}$ it suffices to use terminal grammatical elements only.

6.9. Theorem. Let $\mathscr{L}$ be a language,

$$
H=\{[A, t] ; A \in \mathbf{d} \mathscr{L}, t \in \boldsymbol{\sigma} \mathscr{L}\}, H_{\mathrm{t}}=\left\{[A, t] ; A \in \mathbf{d} \mathscr{L}, t \in \boldsymbol{\sigma}_{\mathrm{t}} \mathscr{L}\right\} .
$$

For every subset $M$ of $H$ and every subset $N$ of $H_{\mathrm{t}}$ we define the closure $\mathrm{Cl} M$ of $M$ and the terminal closure $\mathrm{Cl}_{\mathrm{t}} N$ of $N$ thus:

(1) $\mathrm{Cl} M=M \cup\left\{[A, t] ; A \Rightarrow \alpha, \tau\right.$ is a decomposition, $\lambda \alpha=\lambda \tau, t=\prod \tau$, for every $i \in \mathbf{d} \alpha$ either $[\alpha i]=\tau i$ or $[\alpha i, \tau i] \in M\}$. 
(2) $\mathrm{Cl}_{\mathrm{t}} N=N \cup\left\{[A, t] ; A \Rightarrow \alpha, \tau\right.$ is a decomposition, $\lambda \alpha=\lambda \tau, t=\prod \tau$, for every $i \in \mathbf{d} \alpha$ either $[\alpha i]=\tau i \in \boldsymbol{\sigma}_{\mathrm{t}} \mathscr{L}$ or $\left.[\alpha i, \tau i] \in N\right\}$.

Put

$$
\begin{gathered}
G_{0}=\{[A, t] ;[A] \Rightarrow t\}, \quad Q_{0}=\left\{[A, t] ;[A] \Rightarrow t \in \sigma_{\mathrm{t}} \mathscr{L}\right\}, \\
G_{i}=\mathrm{Cl} G_{i-1}, \quad Q_{i}=\mathrm{Cl}_{\mathrm{t}} Q_{i-1} \text { for } i \in I .
\end{gathered}
$$

Let $G$ and $Q$ be the smallest subsets of $M$ with the properties

$$
\mathrm{Cl} G=G \supset G_{0}, \quad \mathrm{Cl}_{\mathrm{t}} Q=Q \supset Q_{0} .
$$

Then

$$
G=\bigcup_{i=0}^{\infty} G_{i}=\mathbf{g} \mathscr{L}, \quad Q=\bigcup_{i=1}^{\infty} Q_{i}=\mathbf{g}_{\mathrm{t}} \mathscr{L}
$$

Proof. Set $Q_{\infty}=\bigcup_{i=1}^{\infty} Q_{i}$. Applying Lemma. 5.5 we obtain by induction that $Q_{i} \subset$ $\subset Q \subset \mathbf{g}_{\mathrm{t}} \mathscr{L}$ for every $i$, and hence $Q_{\infty} \subset Q \subset \mathbf{g}_{\mathrm{t}} \mathscr{L}$. Note that $\mathrm{Cl}_{\mathrm{t}} Q_{\infty} \subset Q_{\infty}$. Put $M=Q_{\infty} \cup\left(\mathbf{g} \mathscr{L}-\mathbf{g}_{\mathbf{t}} \mathscr{L}\right)$. From (3) we obiain that $[A, t] \in M$ if $[A] \Rightarrow t$. Now if $[A, t] \in \mathbf{g}_{\mathbf{t}} \mathscr{L}$ has an $M$-regular structure $[\alpha, \tau]$, then $[A, t] \in \mathrm{Cl}_{\mathbf{t}} Q_{\infty} \subset Q_{\infty}$ and $[A, t] \in M$. This remains true also if $t$ is not terminal, and an application of Theorem 6.7 yields $M=\mathbf{g} \mathscr{L}$. Hence $Q_{\infty} \supset \mathbf{g}_{\mathrm{t}} \mathscr{L}$ and $Q_{\infty}=Q=\mathbf{g}_{\mathrm{t}} \mathscr{L}$. The first part of (6) may be proved similarly.

6.10. Remark. For some languages it is possible to determine, to every grammatical element $[A, t]$, an integer $n$ such that for every structure $[\alpha, \tau]$ of $[A, t]$ and $i \in \mathbf{d} \alpha$, either $[\alpha i]=\tau i$ or $[\alpha i, \tau i] \in G_{n}$, where $G_{n}$ is the set from the preceding theorem. (For example, if $\lambda \beta \geqq 2$ for every $[B, \beta] \in \mathbf{g} \mathscr{L}$ then it suffices to put $n=\lambda t-2$.) If, moreover, the sets $\mathbf{d} \mathscr{L}$ and $\operatorname{Ur} \mathscr{L}$ are finite, it is possible to find, in a finite number of steps, all the structures of $[A, t]$. In particular it is possible to determine whether or not $[A, t]$ is structurally ambiguous. This procedure, of course, fails to determine whether or not the language is structurally ambiguous, at least if the language has, as it usually does, infinitely many texts.

A similar result follows from the formal power-series reprezentation of N. Chomsky and M. P. Schützenberger [6].

6.11. Example. Let $\mathscr{L}$ be the language defined in Example 5.9. Put $t_{0}=a n$, $t_{1}=a n n n, t_{2}=a 0 n 1, \tau_{1}=[a n n, n], \tau_{2}=[a, 0, n, 1]$. Then the conditions of Lemma 6.1 are satisfied, $\tau=\tau_{1} \otimes \tau_{2}=[a 0 n, 1]$ is a $t_{0}$-decomposition of $t_{2}, \zeta_{1}=$ $=\boldsymbol{\delta}\left(\tau_{2}, \mathbf{\iota} \tau_{1}\right)=[[a, 0, n],[1]]$ and $\zeta_{1} 1=[a, 0, n]$ is a $\tau_{1} 1=$ ann-decomposition of $\tau 1=a 0 n$.

Using the notation of Theorem 6.9 we obtain $Q_{0}=\{[n, 0][n, 1]\}, Q_{1}=\mathrm{Cl}_{\mathrm{t}} Q_{0}=$ $=Q_{0} \cup\{[a, 0],[a, 1]\}, Q_{2}=Q_{1} \cup\{[a, 00],[a, 01],[a, 10],[a, 11]\}, \ldots, \bigcup_{i=0}^{\infty} Q_{i}=$ $=Q_{0} \cup\left\{[a, t] ; t \in \bigcup_{i=1} \mathbf{s}_{i}\{0,1\}\right\}$. 


\section{STRUCTURAL UNAMBIGUITY AND SEMANTICS}

7.1. Definition. We say that a language $\mathscr{L}$ is structurally unambiguous (weakly structurally unambiguous) if every $g \in \mathbf{g} \mathscr{L}$ (every $g \in \mathbf{g}_{\mathfrak{t}} \mathscr{L}$ ) has at most one structure (by Theorem 6.5 it has then exactly one structure).

7.2. Remark. The rest of this section serves to clarify the meaning of (weak) structural unambiguity and the relation of these two concepts. It will be clear that in a certain sense it is superfluous to require structural unambiguity, and that weak structural unambiguity is sufficient. In subsequent sections it will be seen that the intrinsic properties of the former concept are more convenient for study. In the present section it will be proved than in some cases the two concepts coincide.

7.3. Definition. A semantical rule $\Gamma$ is a transformation defined on the set $\{[A, \alpha]$; $[A] \stackrel{\Rightarrow}{=}, A \in \mathbf{d} \mathscr{L}\}$, the values of which are transformations. Denoting $\Gamma[A, \alpha]$ by $\Gamma_{A, \alpha}, R=\bigcup\left\{\mathbf{r} \Gamma_{A, \alpha} ;[A, \alpha] \in \mathbf{d} \Gamma\right\} \cup \mathbf{a}_{\mathrm{t}} \mathscr{L}$, we require that for every $[A, t] \in \mathbf{g}_{\mathrm{t}} \mathscr{L}$ and each of its structures $[\alpha, \tau]$ the following two conditions be satisfied:

$$
\begin{aligned}
& \sigma_{\mathrm{t}} \mathscr{L} \cap \mathscr{L} A \subset \mathbf{d} \Gamma_{A, \alpha} \text { if }[A]=\alpha, \\
& \mathbf{s}_{\lambda \alpha} R \subset \mathbf{d} \Gamma_{A, \alpha} \text { if }[A] \Rightarrow \alpha \notin \boldsymbol{\sigma}_{\mathrm{t}} \mathscr{L} .
\end{aligned}
$$

By a semantics $S$ induced by a semantical rule $\Gamma$ we mean any transformation defined on $\mathbf{g}_{\mathbf{t}} \mathscr{L}$ which satisfies the following conditions:

For every $[A, t] \in \mathbf{g}_{\mathbf{t}} \mathscr{L}$ and every structure $[\alpha, \tau]$ of $[A, t]$ we have

$$
S[A, t]=\Gamma_{A, \alpha} t \quad \text { if } \quad[A]=\alpha
$$

and

$$
\beta \in \mathbf{d} \Gamma_{A, \alpha}, \quad S[A, t]=\Gamma_{A, \alpha} \beta \quad \text { if } \quad[A] \Rightarrow \alpha
$$

where $\beta$ is the sequence of length $\lambda \alpha$ determined by

$$
\beta i=\alpha i \text { if }[\alpha i]=\tau i
$$

and

$$
\beta i=S[\alpha i, \tau i] \text { if }[\alpha i] \neq \tau i .
$$

7.4. Theorem. Every semantical rule induces at most one semantics.

Proof. Let $S_{1}, S_{2}$ be two semantics induced by a semantical rule $\Gamma$, let

$$
M=\left\{g ; g \in \mathbf{g}_{\mathbf{t}} \mathscr{L}, S_{1} g=S_{2} g\right\} \cup\left(\mathbf{g} \mathscr{L}-\mathbf{g}_{\mathbf{t}} \mathscr{L}\right) .
$$

Suppose $[A] \Rightarrow t$. Then either $t$ is not terminal or by (7.3.3) $S_{i}[A, t]=\Gamma_{A,[A]} t$. Hence $[A, t] \in M$. Suppose $[A, t]$ has an $M$-regular structure $[\alpha, \tau]$. If $t$ is not terminal then $[A, t] \in M$. Otherwise $S_{1}[\alpha i, \tau i]=S_{2}[\alpha i, \tau i]$ if $[\alpha i] \neq \tau i$, and by $(7.3 .4)$ we obtain that $S_{1}[A, t]=S_{2}[A, t]$ and thus again $[A, t] \in M$. Application of Theorem 6.7 yields that $M=\mathbf{g} \mathscr{L}$ and $S_{1}=S_{2}$. 
7.5. Theorem. Let $\mathscr{L}$ be a language. Then for the weak structural unambiguity of $\mathscr{L}$ it is sufficient and necessary that every semantical rule $\Gamma$ induce a semantics.

Proof. First let $\mathscr{L}$ be weakly structurally unambiguous, let $\Gamma$ be a semantical rule. Let $Q_{i}=\left\{g ; g \in \mathbf{g}_{\mathbf{t}} \mathscr{L}, \delta g=i\right\}$. We have $\bigcup_{i=1}^{\infty} Q_{i}=\mathbf{g}_{\mathrm{t}} \mathscr{L}$. On $Q_{1}$ we define $S[A, t]=$ $=\Gamma_{A,[A]} t$, which is possible because of (7.3.1). Suppose that $S$ is already defined on $M_{k-1}=\bigcup_{i=1} Q_{i}$ in such a way that (7.3.3) and (7.3.4) hold for every $[A, t]$ in this set (since every $[A, t]$ has only one structure, the grammatical elements $[\alpha i, \tau i]$ in (7.3.4b) are in $M_{k-2}$ according to Theorem 6.5 , and thus $S[\alpha i, \tau i]$ has a meaning); and that, furthermore, $\mathbf{r} S_{M_{k-1}} \subset R$ where $R$ is as in Definition 7.3. Let $[A, t]$ be in $Q_{k}$; we note that this set is disjoint with $M_{k-1}$. Let $[\alpha, \tau]$ be the unique structure of $[A, t]$. We have $[A] \Rightarrow \alpha,[A] \neq \alpha$, and define $\beta$ by $(7.3 .4 \mathrm{a})$ and $(7.3 .4 \mathrm{~b})$. By assumption $\beta \in \mathbf{s}_{\lambda \alpha} R \subset \mathbf{d} \Gamma_{A, \alpha}$ (see 7.3.2). Hence (7.3.3) and (7.3.4) are satisfied if we put $S[A, t]=\Gamma_{A, \alpha} \beta$. Moreover $S[A, t] \in R$. Hence we may complete the definition of $S$ on $M_{k}$, preserving all the properties previously required for $M_{k-1}$. Defining $S$ in this way for all $[A, t]$ in $\mathbf{g} \mathscr{L}$, we obtain that $S$ is a semantics induced by $\Gamma$.

On the other hand, assume that every semantical rule $\Gamma$ induces a semantics. We shall define a $\Gamma$ such that for the semantics $S$ induced by $\Gamma$ we shall have

$S[A, t]=[\alpha, \tau]$ for every $[A, t] \in \mathbf{g}_{\mathrm{t}} \mathscr{L}$ and every structure $[\alpha, \tau]$ of $[A, t]$. This will imply that $\mathscr{L}$ is weakly structurally unambiguous.

First let $N$ be the set of all structures of terminal grammatical elements. We shall suppose that $N$ is disjoint with $\mathbf{a} \mathscr{L}$. (Otherwise $N$ might be replaced by a suitable set $\tilde{N}$ disjoint with a $\mathscr{L}$ and such that there is a one-to-one correspondence between elements of $N$ and $\tilde{N}$.) Further, let $\omega$ be an element which is not in $\mathbf{a} \mathscr{L} \cup N$, let

$$
H=\mathbf{s}\left(\mathbf{a}_{\mathbf{t}} \mathscr{L} \cup N \cup\{\omega\}\right), \quad H_{0}=\mathbf{s}\left(\mathbf{a}_{\mathbf{t}} \mathscr{L} \cup N\right) .
$$

Define $\psi$ on $\mathbf{a}_{\mathrm{t}} \mathscr{L} \cup N$ by

$$
\psi a=[a] \text { for } a \in \mathbf{a}_{\mathbf{t}} \mathscr{L}, \quad \psi[\alpha, \tau]=\prod \tau \text { for }[\alpha, \tau] \in N .
$$

For $a \in H_{0}$ define $\varphi a$ as the sequence for which

$$
\lambda \varphi a=\lambda a, \quad(\varphi a) i=\psi a i \text { for } i \in \mathbf{d} a .
$$

Now for $A \in \mathbf{d} \mathscr{L}, t \in \mathscr{L} A, t$ terminal we define

$$
\Gamma_{\mathrm{A},[\mathrm{A}]} t=[[A],[t]] ;
$$

for $A \in \mathrm{d} \mathscr{L}, \alpha \in \mathscr{L} A, \beta \in H, \lambda \beta=\lambda \alpha$ we put

(5) if $\beta \in H_{0},[\alpha, \varphi \beta] \in N$ 
then

$$
\Gamma_{\mathrm{A}, \alpha} \beta=[\alpha, \varphi \beta]
$$

else

$$
\Gamma_{\mathrm{A}, \alpha} \beta=\omega \text {. }
$$

According to this definition, $\mathbf{r} \Gamma_{\mathrm{A}, \alpha} \subset\{\omega\} \cup N$ for every $A \in \mathbf{d} \mathscr{L},[A] \rightrightarrows \alpha$. The set $R$ from Definition 7.3 is $\{\omega\} \cup N \cup \mathbf{a}_{\mathbf{t}} \mathscr{L}, s_{\lambda \alpha} R=\{\beta ; \beta \in H, \lambda \beta=\lambda \alpha\}$ and both (7.3.1) and (7.3.2) are satisfied.

Hence $\Gamma$ is a semantical rule. Let $S$ be induced by $\Gamma$. We have to prove that

(6) if $[A, t] \in \mathbf{g}_{\mathrm{t}} \mathscr{L}$ and $[\alpha, \tau]$ is a structure of $[A, t]$ then $S[A, t]=[\alpha, \tau]$.

This property implies

$$
\text { if }[A, t] \in \mathbf{g}_{\mathbf{t}} \mathscr{L} \text { then } S[A, t] \in N \text { and } \psi S[A, t]=t \text {. }
$$

Let $M$ be the set of all such $[A, t] \in \mathbf{g} \mathscr{L}$ for which (7) holds. Let $[A, t] \in \mathbf{g}_{\mathbf{t}} \mathscr{L}$, let $[\alpha, \tau]$ be a structure of $[A, t]$.

If $[A]=\alpha$ then by (5.7.3a) $\tau=[t]$ and by (7.3.3) and (4) $S[A, t]=\Gamma_{\mathrm{A},[\mathrm{A}]} t=$ $=[\alpha, \tau]$ and (6) holds. Hence, in particular, if $A \Rightarrow t,[A, t] \in M$. Now let $[A] \neq \alpha$ but let

$$
[\alpha, \tau] \text { be weakly } M \text {-regular . }
$$

For every $i \in \mathbf{d} \alpha$ either $[\alpha i]=\tau i$ and, by (2), $\psi \alpha i=\tau i$, or $[\alpha i] \neq \tau i$ and, by (7), $\psi S[\alpha i, \tau i]=\tau i$. Hence if $\beta$ is defined as in (7.3.4), then we obtain, according to (3), that $(\varphi \beta) i=\tau i$ for every $i \in \mathbf{d} \alpha$, i.e. that $\varphi \beta=\tau$. By (7.3.4) and (5) we obtain $S[A, t]=\Gamma_{\mathrm{A}, \alpha} \beta=[\alpha, \tau]$, and (6) holds for our $A, t, \alpha, \tau$. In particular, $[A, t] \in M$ and, by Theorem $6.7, M=\mathbf{g} \mathscr{L}$. The assumption (8) is satisfied for every structure and (6) holds for every $[A, t]$ and the proof is completed.

7.6. Theorem. Let $\mathscr{L}$ be structurally unambiguous. Then $\alpha \rightarrow \alpha$ for no $\alpha \in \cup \mathbf{r} \mathscr{L}$.

Proof. Let $\alpha \in \mathscr{L} A, A \in \mathbf{d} \mathscr{L}, \alpha \rightarrow \alpha$. Then there is an $\alpha$-decomposition $\tau$ of $\alpha$ and $[A, \alpha]$ has two structures $[[A],[\alpha]]$ and $[\alpha, \tau]$ which are distinct since $[A] \neq \alpha$ by (5.2.2).

7.7. Theorem. Let $\mathscr{L}$ be a language satisfying the following conditions:

$$
\begin{gathered}
\mathbf{t}_{\mathbf{t}}(\mathscr{L}, A)-\{\Lambda\} \neq \Lambda \text { for every } A \in \mathbf{d} \mathscr{L}, \\
\alpha \rightarrow \alpha \text { for no } \alpha \in \operatorname{Ur} \mathscr{L} .
\end{gathered}
$$

Then $\mathscr{L}$ is structurally unambiguous if and only if it is weakly structurally unambiguous.

Proof. Suppose that $g=[A, t] \in \mathbf{g} \mathscr{L}-\mathbf{g}_{\mathrm{t}} \mathscr{L}$, that $\left[\alpha_{1}, \tau_{1}\right],\left[\alpha_{2}, \tau_{2}\right]$ are two different structures of $g$, and that $\mathscr{L}$ is weakly structurally unambiguous. For the proof 
it suffices to prove that this assumption leads to a contradiction. For every $i \in \mathbf{d} t$ let us choose a terminal string $u_{i}$ such that $[t i] \rightrightarrows u_{i} \neq \Lambda$. Let $\lambda \xi=\lambda t, \xi i=u_{i}$ for $i \in \mathbf{d} t$, $u=\prod \xi$. We see that $\xi$ is a $t$-decomposition of $u, t \rightarrow u,[A, u] \in \mathbf{g}_{\mathbf{t}} \mathscr{L}$.

By Lemma 6.1, $\tau_{i} \otimes \xi$ is an $\alpha_{i}$-decomposition of $u$. By (5.7.3a) and (5.7.3b) we have for both $i=1,2$ either

$$
[A]=\alpha_{i} \Rightarrow t \text { and }[t, \xi] \text { is a structure of }[A, u]
$$

or

$$
[A] \Rightarrow \alpha_{i} \rightarrow t \rightarrow u,\left[\alpha_{i}, \tau_{i} \otimes \xi\right] \text { is a structure of }[A, u] .
$$

Suppose (4) holds for both $i=1,2$. But then $\left[\alpha_{i}, \tau_{i}\right]=[[A],[t]]$ for $i=1,2$ which contradicts the assumption that $\left[\alpha_{1}, \tau_{1}\right] \neq\left[\alpha_{2}, \tau_{2}\right]$. Suppose (4) holds for $i=1,(5)$ holds for $i=2$. Then $[A] \Rightarrow \alpha_{2} \rightarrow t$ and by $(2) \alpha_{2} \neq t$. Hence $[t, \xi]$, $\left[\alpha_{2}, \tau_{2} \otimes \xi\right]$ are distinct structures of $[A, u]$ which contradicts the assumption. A similar result can be obtained if (6) holds for $i=2$ and (5) holds for $i=1$. Thus we may assume that (5) holds for $i=1,2$. Then $\left[\alpha_{1}, \tau_{1} \otimes \xi\right]=\left[\alpha_{2}, \tau_{2} \otimes \xi\right]$. We conclude immediately that $\alpha_{1}=\alpha_{2}$, and from Lemma 4.11 that $\tau_{1}=\tau_{2}$ which is the desired contradiction.

7.8. Example. Consider the language $\mathscr{L}$ from Examples 5.9 and 6.11. Any semantical rule $\Gamma$ for $\mathscr{L}$ must be defined on the set $M=\{[a, a],[a, n],[a, a n],[n, n]$, $[n, 0],[n, 1]\}$. Let $\Gamma_{a, a}=\Gamma_{n, 0}=\Gamma_{n, 1}=\Lambda, \Gamma_{a, n}$ and $\Gamma_{n n}$ be the identical transformations on $\{0,1\}$ and let $\Gamma_{a, a n}$ be defined on $\mathbf{s}_{2} I_{0}$ by the relation $\Gamma_{a, a n} \beta=2 \times \beta 1+$ $+\beta 2$ where $\times$ and + denote the multiplication and addition of numbers respectively. Then $\Gamma$ is a semantical rule and induces exactly one semantics $S$. The value $S[a, t]$ for $t \in \mathbf{t}_{\mathbf{t}}(\mathscr{L}, a)$ is clearly the number in $I_{0}$ with binary representation $t$.

\section{RELATIONS BETWEEN LANGUAGES}

8.1. Lemma. Let $\mathscr{L}$ be a language, $\mathscr{L}_{0} \subset \mathscr{L}$. Then $\mathscr{L}_{0}$ is a language and the relations $\mathscr{L}_{0}: \Rightarrow, \mathscr{L}_{0} \rightarrow,: \mathscr{L}_{0}: \rightarrow$ are stronger than the relations $\mathscr{L}: \Rightarrow, \mathscr{L}: \rightarrow$, $\mathscr{L}: \rightarrow$, respectively, i.e. $\mathscr{L}_{0}: a \Rightarrow b$ implies $\mathscr{L}: a \Rightarrow b$ and so on.

Proof. Any subset of a transformation is again a transformation; so is $\mathscr{L}_{0}$. It is easy to verify that conditions (5.2.1) and (5.2.2) hold with $\mathbf{A}=\mathbf{a} \mathscr{L}$, and that the relations $\Rightarrow, \rightarrow, \rightarrow$ with respect to $\mathscr{L}_{0}$ are stronger than those with respect to $\mathscr{L}$.

8.2. Lemma. Let $\mathscr{L}$ be a language, $\mathscr{L}_{0} \subset \mathscr{L}$. Then $\mathbf{g} \mathscr{L}_{0} \subset \mathbf{g} \mathscr{L}$, and if $g \in \mathbf{g} \mathscr{L}_{0}$ and $[\alpha, \tau]$ is a structure of $g$ in $\mathscr{L}_{0}$ then $[\alpha, \tau]$ is also a structure of $g$ in $\mathscr{L}$.

Proof. Straightforward from Definition 5.7.

8.3. Lemma. Let $\mathscr{L}$ be a structurally unambiguous language, $\mathscr{L}_{0} \subset \mathscr{L}$. Then $\mathscr{L}_{0}$ is structurally unambiguous.

Proof. From Lemma 8.2. 


\section{REDUCING PAIRS AND REDUCING TRANSFORMATIONS}

9.1. Definition. A reducing pair is a pair $\langle V, R\rangle$ of transformations $V$ and $R$ defined on $\mathbf{g} \mathscr{L}$ such that for every $g=[A, t] \in \mathbf{g} \mathscr{L}$, every structure $[\alpha, \tau]$ of $g$ and for $\varrho g$ defined as $[A, V g]$ we have:

(1) $R g$ is a $V g$-decomposition of $t$.

(2) If $t \neq V g=[A]$ then $\alpha=[A]$.

(3) If $[A] \Rightarrow t$ then $V g \in\{[A], t\}$.

(4) There is an $\alpha$-decomposition $\xi$ of $V g$ such that $\tau=\xi \otimes R g$.

(5) If $g_{i} \in \mathbf{g} \mathscr{L}, \varrho g_{i}=g_{i+1}$ for $i=1,2, \ldots$ then there is a $j$ such that $g_{j}$ is $\varrho$-invariant, i.e. $\varrho g_{j}=g_{j}$.

(6) If $\varrho g=g, i \in \mathbf{d} \alpha,[\alpha i] \neq \tau i$ then $\varrho[\alpha i, \tau i]=[\alpha i, \tau i]$.

The transformation $\varrho$ defined on $\mathbf{g} \mathscr{L}$ is termed reducing (and induced by $\langle V, R\rangle$ ). If not all conditions (1)-(6) are required but only conditions (1)-(i) we speak about an (i)-reducing pair or transformation.

9.2. Remark. The concept of a reducing pair may be motivated as follows. Suppose $[A, t]$ is a grammatical element. Sometimes we may recognize a part $t^{(i, j)}$ of the text $t$ as being necessarily derived from a metasymbol $B$. Then we may define a new text $V g=t^{(1, i-1)} \times[B] \times t^{(j+1, \lambda t)}$ and a decomposition $R g=\delta_{p}\left(t^{(1, i+1)}\right) \times\left[t^{(i, j)}\right] \times$ $\times \boldsymbol{\delta}_{p}\left(t^{(j+1, \lambda t)}\right)$. For every $i \in \mathbf{d} V g$ the decomposition $R g$ determines a part $(R g) i$ of $t$ which may be derived from $[(V g) i]$. Under some conditions it can be proved that if $[A, V g]$ is a grammatical element then it has the same number of structures as $[A, t]$. These conditions are given in (1) to (4). Conditions (5) and (6) require some further regularity properties which are used to derive stronger results in Theorems 9.4, 9.5, 9.11 and 9.13. On the other hand the conditions in this definition are not unnecessarily strong. In fact, if $\mathscr{L}$ is structurally unambiguous, and $[V g, R g]$ is the unique structure of $g \in \mathbf{g} \mathscr{L}$ then it is easily verified that $\langle V, R\rangle$ is a reducing pair.

9.3. Lemma. Let $\varrho$ be a reducing (or (4)-reducing) transformation, let $g \in \mathbf{g} \mathscr{L}$, and either $\varrho g \notin \mathbf{g} \mathscr{L}$ or $\varrho g$ be structurally unambiguous. Then $g$ is also structurally unambiguous.

Proof. Let $\langle V, R\rangle$ induce $\varrho$, let $\left[\alpha_{i}, \tau_{i}\right](i=1,2)$ be two different structures of a $g \in \mathbf{g} \mathscr{L}, \varrho g \notin \mathbf{g} \mathscr{L}$ or $\varrho g$ be structurally unambiguous. Then we have $\varrho g \neq g$. By (9.1.4) there are $\alpha_{i}$-decompositions $\xi_{i}$ of $V g$ such that

$$
\tau_{i}=\xi_{i} \otimes R g
$$

and

$$
[A] \stackrel{\Rightarrow}{\equiv} \alpha_{i} \rightrightarrows V g
$$


First suppose $[A]=V g$. In this case (9.1.2) implies $\alpha_{i}=[A]$; hence $\tau_{i}=[t]$, which contradicts the assumption $\left[\alpha_{1}, \tau_{1}\right] \neq\left[\alpha_{2}, \tau_{2}\right]$. Hence $[A] \neq V g$ and by (9.1.3)

$$
[A] \Rightarrow \alpha_{1}, \quad[A] \Rightarrow \alpha_{2} \text {. }
$$

Now suppose $V g \in \mathscr{L} A$. Then $\varrho g$ has a structure [[A], $[V g]]$. If, for some $i, \alpha_{i} \neq$ $\neq V g$ then $\alpha_{i} \rightarrow V g$ and $\varrho g$ has a structure $\left[\alpha_{i}, \xi_{i}\right]$. By (5.2.2) and (3), $\alpha_{i} \neq[A]$, and $\varrho g$ has two different structures, which is impossible. Hence $V g=\alpha_{1}=\alpha_{2}$. Suppose $\tau_{i} \neq R g$ for some $i$. Then $\xi_{i}$ is not primitive and, since $\xi_{i}$ is an $\alpha_{i}$-decomposition of $V g=\alpha_{i}$, we have $\alpha_{i} \rightarrow \alpha_{i}$ and $\varrho g=\left[A, \alpha_{i}\right]$ has two different structures $[[A],[\alpha i]]$, $\left[\alpha_{i}, \xi_{i}\right]$, which again is impossible. Hence $V g \in \mathscr{L} A$ implies $\alpha_{1}=\alpha_{2}=V g, \tau_{1}=$ $=\tau_{2}=R g$ which shows that $V g \notin \mathscr{L A}$ and we obtain

$$
\alpha_{1} \rightarrow V g, \quad \alpha_{2} \rightarrow V g
$$

and $\left[\alpha_{1}, \xi_{1}\right],\left[\alpha_{2}, \xi_{2}\right]$ are structures of $\varrho g$. Hence $\left[\alpha_{1}, \xi_{1}\right]=\left[\alpha_{2}, \xi_{2}\right]$ and by $(1)$ $\left[\alpha_{1}, \tau_{1}\right]=\left[\alpha_{2}, \tau_{2}\right]$. This contradiction completes the proof.

9.4. Theorem. Let $\varrho$ be a reducing or ((5)-reducing) transformation. Then $\mathscr{L}$ is structurally unambiguous if and only if every $\varrho$-invariant (i.e. such that $\varrho g=g$ ) grammatical element is structurally unambiguous.

Proof. By (9.1.5) and Lemma 9.3.

9.5. Theorem. The existence of a reducing transformation @ such that no grammatical element is $Q$-invariant is a necessary and sufficient condition for the structural unambiguity of $\mathscr{L}$.

Proof. The sufficiency follows from Theorem 9.3. If $\mathscr{L}$ is structurally unambiguous and $[V g, R g]$ is the unique structure of $g$ for every $g \in \mathbf{g} \mathscr{L}$ then it is easy to verify that $\langle V, R\rangle$ is a reducing pair. Let $\varrho$ be induced by $\langle V, R\rangle, g \in \mathbf{g} \mathscr{L}, \varrho g=g=$ $=[A, t]$. Then $g$ has a structure $[\alpha, \tau]$ with $\alpha=t$, which implies that either $[A] \Rightarrow$ $\Rightarrow t \rightarrow t$ or $[A]=t \Rightarrow t$. The former case is impossible according to Theorem 7.6, the latter case is impossible according to (5.2.2). Hence no grammatical element is $\varrho$-invariant.

9.6. Theorem. Let $V, R$ be two transformations defined on $\mathbf{g} \mathscr{L}$. For every $g=$ $=[A, t] \in \mathbf{g} \mathscr{L}$ let the following two conditions hold:

(1) $R g$ is a decomposition of $t, V g$ is a sequence, $\lambda V g=\lambda R g$.

(2) For every structure $[\alpha, \tau]$ of $g$ there is an index-decomposition $x_{0}$ of $V g$ such that the decompositions $\xi=\delta\left(V g, x_{0}\right)$ and $\zeta=\delta\left(R g, x_{0}\right)$ satisfy

$$
\tau=\zeta \otimes R g
$$


and, for every $i \in \mathbf{d} \alpha$, at least one of conditions (2b1), (2b2) and (2b3) holds:

$$
\begin{gathered}
\lambda \xi i=1, \quad[\alpha i] \rightrightarrows \xi i \rightarrow \tau i, \quad \delta_{0}[\alpha i, \xi i]<\delta_{0}[\alpha i, \tau i], \\
{[A] \neq \alpha, \quad[\alpha i] \neq \tau i, \quad \xi i=V[\alpha i, \tau i], \quad \zeta i=R[\alpha i, \tau i],} \\
\xi i=\tau i, \quad \zeta i=\delta_{p}(\tau i) .
\end{gathered}
$$

Then conditions (9.1.1), (9.1.3) and (9.1.4) hold for every $g \in \mathbf{g} \mathscr{L}$ and each of its structures $[\alpha, \tau]$. Moreover if for every such $g=[A, t]$ and $[\alpha, \tau]$ there holds (9.1.2), then condition (9.1.5) is satisfied.

Hence if conditions (9.1.2) and (9.1.6) are satisfied, $\langle V, R\rangle$ is a reducing pair.

Proof. First prove that $(9.1 .3)$ holds. Let $[A] \Rightarrow t,[\alpha, \tau]=[[A],[t]]$. Then the decompositions $\xi$ and $\zeta$ satisfy $\lambda \xi=\lambda \zeta=\lambda \tau=1$ because of (2a) and at least one of conditions (2b1), (2b2) and (2b3) is satisfied for $i=1$. (2b2) does not hold. If (2b1) holds then $\delta_{0}[A, V g]<\delta_{0}[A, t]=1$, whence $A=V g$. If $(2 \mathrm{~b} 3)$ holds then $V g=t$. Hence (9.1.3) holds.

Secondly we shall show that conditions (1) and (2) imply (9.1.1) and (9.1.4). Concerning the latter condition if suffices to prove that $\xi$ is an $\alpha$-decomposition of $V g$ (we note that $\xi \otimes R g=\zeta \otimes R g=\tau$ ). Since $\xi$ is a decomposition of $V g$ we have to prove that

$$
[\alpha i] \rightrightarrows \xi i, \quad[(V g) j] \rightrightarrows(R g) j \quad \text { for every } \quad i \in \mathbf{d} \alpha, j \in \mathbf{d} V g .
$$

We note that (3) implies the following property which is independent of $[\alpha, \tau]$ :

$$
[A] \rightrightarrows V g, R g \text { is a } V g \text {-decomposition of } t \text {. }
$$

In proving (3) we may assume $x_{0} i \leqq j<x_{0}(i+1)$. Put $i_{0}=x_{0} i, i_{1}=x_{0}(i+1)-$ - 1. Note that according to (4.4.5) we have $\xi i=(V g)^{\left(i_{0}, i_{1}\right)}, \zeta i=(R g)^{\left(i_{0}, i_{1}\right)}$, according to (4.2.5) we obtain $(V g) j=(\xi i)\left(j+1-i_{0}\right),(R g) j=(\zeta i)\left(j+1-i_{0}\right)$. From (2a) and Lemma (4.8) we have $\tau i=\prod \zeta i$. We shall show that each of the two conditions (2b1), (2b3) implies (3). The condition $[\alpha i] \rightrightarrows \xi i$ is explicitly expressed in (2b1) and follows from the relation $[\alpha i] \rightrightarrows \tau i$ if $(2 \mathrm{~b} 3)$ holds. It remains to prove $[(V g) j] \rightrightarrows(R g) j$. If $(2 \mathrm{~b} 1)$ holds then $\lambda \xi i=1$ which implies $i_{0}=j=i_{1}$ and $[(V g) j]=[(\xi i) 1]=\xi i \rightarrow \tau i=\prod \zeta i=(\zeta i) 1=(R g) j$. If $(2 \mathrm{~b} 3)$ holds then $[(V g) j]=\left[(\xi i)\left(j+1-i_{0}\right)\right]=\left[(\tau i)\left(j+1-i_{0}\right)\right]=$ $=\left(\delta_{p}(\tau i)\right)\left(j+1-i_{0}\right)=(\zeta i)\left(j+1-i_{0}\right)=(R g) j$. Hence if $(2 \mathrm{~b} 1)$ or $(2 \mathrm{~b} 3)$ then (3) holds. Now let $M$ be the set of all such $g$ in $\mathbf{g} \mathscr{L}$ for which (4) holds. Let $[A] \Rightarrow t$. Then $[\alpha, \tau]=[[A],[t]]$ is a structure of $[A, t]$. By (2), applied for $i=1$, we have that either (2b1) or (2b3) holds. Hence (3) and (4) hold for our $g=[A, t]$ and $g \in M$.

Suppose that $g=[A, t]$ has a weakly $M$-regular structure $[\alpha, \tau]$. We shall prove that in this case (3) holds. Let $i \in \mathbf{d} \alpha$ be given. If (2b1) or (2b3) hold, (3) is satisfied. 
Let (2b2) hold. Then $[\alpha i, \tau i] \in M$ and by (4) applied for $[\alpha i, \tau i]$ we get from (2b2) that $[\alpha i] \rightrightarrows \xi i, \zeta i$ is a $\xi i$-decomposition of $\tau i$. Hence, putting $j_{0}=j-x_{0} i+1$, we have $[(V g) j]=\left[(\xi i) j_{0}\right] \rightrightarrows(\zeta i) j_{0}=(R g) j$ and conditions (3) and (4) hold for our $g$. Hence $g \in M$. By Theorem $6.7, M=\mathbf{g} \mathscr{L}$. Thus every structure $[\alpha, \tau]$ of a grammatical element $g$ is weakly $M$-regular and (3) holds for every $g \in \mathbf{g} \mathscr{L}$ and each of its structures $[\alpha, \tau]$, which completes the proof of (9.1.1) and (9.1.4).

Now suppose that (9.1.2) holds and let us prove that the set $M=\{g ; g=[A, t] \in$ $\in \mathbf{g} \mathscr{L}$ and $(V g=[A]$ or $V g=t$ or $\delta \varrho g<\delta g)\}$ is equal to $\mathbf{g} \mathscr{L}$. This will imply that condition (9.1.5) holds, since $\delta$ is positive-integer-valued and $t \neq V g=[A]$ implies $\varrho g \notin \mathbf{g} \mathscr{L}$. The last implication can be proved as follows. If $V g=[A] \neq t$ and $[A] \rightarrow$ $\rightarrow[A]$ then there is a structure $[\alpha, \tau]$ of $[A, t]$ such that $\alpha \neq[A]$, which contra$\operatorname{dicts}(9.1 .2)$.

Now let $[A] \Rightarrow t$. Then by $(9.1 .3)[A, t] \in M$. Let $[\alpha, \tau]$ be an $M$-regular structure of $g=[A, t]$, let $t \neq V g \neq[A]$ and not $[A] \Rightarrow t$. Let $x_{0}$, $\xi$ and $\zeta$ be as in (2). We have $\delta_{0}[\alpha i, \xi i]<\delta_{0}[\alpha i, \tau i]$ if $(2 \mathrm{~b} 1)$ holds. The same inequality follows from the $M$-regularity if $(2 \mathrm{~b} 2)$ holds and $\xi i \neq \tau i$. At least for one $i$ one of these conditions is satisfied because otherwise we would have $V g=\prod \tau=t$, contrary to the assumption. Since for every $i$ we have $\delta_{0}[\alpha i, \xi i] \leqq \delta_{0}[\alpha i, \tau i]$ and since $\xi$ is an $\alpha$-decomposition of $V g$ we obtain by (6.4.1) and (6.6.1)

$$
\delta \varrho g \leqq \delta[A, \alpha]+\sum_{i=1}^{\lambda t_{1}} \delta_{0}[\alpha i, \xi i]<1+\sum_{i=1}^{\lambda t_{1}} \delta_{0}[\alpha i, \tau i]=\delta g
$$

and $g \in M$. By Theorem 6.7 this implies $M=\mathbf{g} \mathscr{L}$.

9.7. Definition. (This definition is motivated by Theorem 9.4 which states that if $\varrho$ is a reducing transformation then it suffices to investigate, for structural unambiguity, only $\varrho$-invariant grammatical elements. If then, for given $A, \alpha, i$, every structure $[\alpha, \tau]$ of such an element $[A, t]$ satisfies $[\alpha i]=\tau i$, we may attempt to replace $\alpha i$ in $\alpha$ by another, terminal, element. An isolating transformation $\omega$ then determines for which $A, \alpha, i$ such a replacement should be made. (The "strongest" $\omega$ for a given $\varrho$ will be such that $\omega[A, \alpha, i] \neq \alpha i$ if and only if $[\alpha i]=\tau i$ for every $\alpha, \tau$ as above. However it suffices and is more convenient to require only the one-sided condition (3).) The function $\varphi$ then determines the element $\varphi \alpha i$ by which the original $\alpha i$ may be replaced. Condition (2.3) is not essential but ensures that $\varphi a$ determines a new terminal element only if such a new element is required. The new language $\mathscr{L}_{0}$ is then defined: If $\alpha \in \mathscr{L} A$ then $\mathscr{L}_{0} A$ contains $\alpha_{0}$ which we get by isolating (i.e. replacing by $\varphi \propto i)$ those elements of $\alpha$ for which $\omega[A, \alpha, i] \neq \alpha i$. Moreover, $\mathscr{L}_{0} A$ contains all other $\alpha_{1}$ obtained by isolating some other symbols $\alpha i$ such that $\varphi \alpha i \neq \alpha i$.)

$\omega$ is an isolating transformation for a language $\mathscr{L}$ if the following conditions are satisfied:

$$
\mathbf{d} \omega=\{[A, \alpha, i] ;[A] \Rightarrow \alpha, i \in \mathbf{d} \alpha\} .
$$


(2) There is a one-to-one transformation $\varphi$ on $\mathbf{a} \mathscr{L}$ such that

(2.1) for every $a \in \mathbf{a} \mathscr{L}$ either $\varphi a=a$ or $\varphi a \notin \mathbf{a} \mathscr{L}$,

(2.2) $\omega[A, \alpha, i] \in\{\alpha i, \varphi \alpha i\}$ for every $[A, \alpha, i] \in \mathbf{d} \omega$

and

(2.3) $\varphi a \neq a$ only if there is an $[A, \alpha, i] \in \mathbf{d} \omega$ such that $\omega[A, \alpha, i] \neq \alpha i=a$.

(3) There is a reducing transformation $\varrho$ such that if $[\alpha, \tau]$ is a structure of a $\varrho$-invariant grammatical element $[A, t]$ and if $[A, \alpha, i] \in \mathbf{d} \omega, \omega[A, \alpha, i] \neq \alpha i$ then $[\alpha i]=\tau i$.

If $\omega$ is an isolating transformation, $\mathscr{A} \subset \mathbf{d} \mathscr{L}$ and $\omega[A, \alpha, i] \neq \alpha i$ for every $[A, \alpha, i] \in \mathbf{d} \omega$ such that $\alpha i \in \mathscr{A}$ then we say that $\mathscr{A}$ is isolable (by $\omega$ ).

We remark that according to (2.3) the transformation $\varphi$ is uniquely determined by $\omega$. We say that $\mathscr{L}_{0}$ is an isolation of $\mathscr{L}$ (by $\left.\omega\right)$ if $\omega$ is an isolating transformation for $\mathscr{L}$ and if

$$
\begin{gathered}
\mathbf{d} \mathscr{L}_{0}=\mathbf{d} \mathscr{L}, \\
\mathscr{L}_{0} A=\text { Is }\{\psi[A, \alpha] ; \alpha \in \mathscr{L} A\},
\end{gathered}
$$

where

$$
\psi[A, \alpha]=\prod_{i=1}^{\lambda \alpha}[\omega[A, \alpha, i]]
$$

and where, for any set $M$ of texts,

(7) Is $M=\left\{t ; t_{0} \in M, \lambda t=\lambda t_{0}\right.$ and for every $i \in \mathbf{d} t$ either $t i=t_{0} i$ or $\left.t i=\varphi t_{0} i\right\}$.

It is easy to see that $\mathscr{L}_{0}$ is a language,

$$
\mathbf{a} \mathscr{L}_{0} \subset \mathbf{a} \mathscr{L} \cup \mathbf{r} \varphi
$$

At the end of this section we shall prove three theorems in which the properties of $\mathscr{L}$ are compared with those of $\mathscr{L}_{0}$ or of another language $\mathscr{L}_{1}$ related to $\mathscr{L}_{0}$. These are preceded by some lemmas in which the following conventions are used (the reader who is interested only in results may skip the following text up to Theorem 9.11):

First, for every $t_{0} \in \sigma \mathscr{L}_{0}$ there is exactly one element $v t_{0}$ in $\sigma \mathscr{L}$ for which

$$
\lambda v t_{0}=\lambda t_{0}, t_{0} i \in\left\{\left(v t_{0}\right) i, \varphi\left(v t_{0}\right) i\right\} \text { for every } i \in \mathbf{d} t_{0} .
$$

Further we write, for $\tau_{0}$ in $\mathbf{s} \boldsymbol{\sigma} \mathscr{L}_{0}, \mu \tau_{0}=\prod_{i=1}^{\lambda \tau_{0}}\left[v \tau_{0} i\right]$; we have $\mu \tau_{0} \in \mathbf{s} \boldsymbol{\sigma} \mathscr{L}$.

On the other hand to every $t$ in $\sigma \mathscr{L}$ we make correspond the sequence $v_{0} t$ of the same length as $t$ defined as $\prod_{i=1}^{\lambda t}\left[\varphi v_{0} t\right]$. For every $\tau$ in $\mathbf{s} \boldsymbol{\mathscr { L }}$ we define $\mu_{0} \tau$ as $\prod_{i=1}^{\lambda \tau}\left[v_{0} \tau i\right]$. 


\subsection{Lemma.}

(1) The relations $\Rightarrow, \rightarrow, \rightarrow, \Rightarrow, \rightrightarrows$, $\rightrightarrows$ are stronger in $\mathscr{L}_{0}$ than in $\mathscr{L}$ in the sense that $\mathscr{L}_{0}: t_{1} \Rightarrow t_{2}$ implies $\mathscr{L}: v t_{1} \Rightarrow v t_{2}$ etc.

(2) If $[A, t] \in \mathbf{g} \mathscr{L}_{0}$ then $[A, v t] \in \mathbf{g} \mathscr{L}$.

(3) If $[\alpha, \tau]$ is a structure in $\mathscr{L}_{0}$ of a grammatical element $[A, t]$ of $\mathscr{L}_{0}$ then $[\nu \alpha, \mu \tau]$ is a structure in $\mathscr{L}$ of $[A, v t]$.

Proof. Let $\alpha \in \mathscr{L}_{0} A, A \in \mathbf{d} \mathscr{L}_{0}$. Then $A \in \mathbf{d} \mathscr{L}_{0}=\mathbf{d} \mathscr{L}, \alpha \in \mathbf{I s}\left\{\psi\left[A, \alpha_{1}\right]\right\}$ for some $\alpha_{1} \in \mathscr{L} A, v \alpha=\alpha_{1}, v[A]=[A]$. Hence $\Rightarrow$ is stronger in $\mathscr{L}_{0}$ than in $\mathscr{L}$. The rest of (1) follows easily, and (1) implies (2) and (3).

9.9. Lemma. Let $g=[A, t]$ be a @-invariant grammatical element of $\mathscr{L}$. Then $g_{0}=\left[A, v_{0} t\right]$ is a grammatical element of $\mathscr{L}_{0}$. Moreover if $[\alpha, \tau]$ is a structure of $g$ and if $\lambda \alpha_{0}=\lambda \alpha, \alpha_{0} i=\alpha i$ whenever $[\alpha i] \neq \tau i$ and $\alpha_{0} i=\varphi \alpha i$ otherwise, then $\left[\alpha_{0}, \mu_{0} \tau\right]$ is a structure of $g_{0}$ in $\mathscr{L}_{0}$.

Proof. Let $M$ be the set of all $g \in \mathbf{g} \mathscr{L}$ which either are not $\varrho$-ivariant or for which the first assertion of the theorem holds.

Let $g=[A, t]$ be $\varrho$-invariant, let $[\alpha, \tau]$ be a structure in $\mathscr{L}$ of $g$.

First suppose $\alpha=[A]$. Then $t \in \mathscr{L} A, v_{0} t \in \mathscr{L}_{0} A$, i.e. $g \in M$. Also $\lambda \alpha_{0}=1$, and $\alpha_{0} 1=\alpha 1=A$ since $[A] \neq t . \mu_{0} \tau=\mu_{0}[t]=\left[v_{0} t\right]$ and $\left[\alpha_{0}, \mu \tau_{0}\right]=\left[[A],\left[v_{0} t\right]\right]$ is. a structure in $\mathscr{L}_{0}$ of $g_{0}$. Hence in this case the assertion of the lemma holds. In particular, $g \in M$ if $t \in \mathscr{L} A$.

Now let $\alpha \neq[A]$ but suppose in addition that $[\alpha, \tau]$ is weakly $M$-regular. If $[\alpha i]=\tau i$ then $\left[\alpha_{0} i\right]=[\varphi \alpha i]=v_{0}[\alpha i]=v_{0} \tau i=\left(\mu_{0} \tau\right) i$. If $[\alpha i] \neq \tau i$ then $\alpha_{0} i=\alpha i$, $[\alpha i, \tau i]$ is $\varrho$-invariant (see (9.1.6)) and by weak $M$-regularity, $\left[\alpha_{0} i, v_{0} \tau i\right] \in \mathbf{g} \mathscr{L}_{0}$. In this case we also have $\omega[A, \alpha, i]=\alpha i$ (by (9.7.3)) which shows that $\alpha_{0} \in \mathbf{I s}\{\psi[A, \alpha]\} \subset$ $\subset \mathscr{L}_{0} A$. Hence $\left[A, t_{0}\right]$ is in $\mathbf{g} \mathscr{L}_{0}$ and $\left[\alpha_{0}, \mu_{0} \tau\right]$ is its structure. In particular, $g \in M$, by Theorem $6.7 M=\mathbf{g} \mathscr{L}$ and every structure $[\alpha, \tau]$ in $\mathscr{L}$ is weakly $M$-regular. This completes the proof.

9.10. Lemma. Let $\mathscr{A}$ be a subset of $\mathbf{d} \mathscr{L}$ isolable by $\omega$, let $\mathscr{L}_{1}=\mathscr{L}_{\mathbf{d} \mathscr{L}-\mathscr{A}}$. Let $[A, t] \in \mathbf{g} \mathscr{L}_{0}$.

Then:

(1) If $A \notin \mathscr{A}$ then $v t \in \mathbf{t}\left(\mathscr{L}_{1}, A\right)$.

(2) If $[\alpha, \tau]$ is a structure in $\mathscr{L}_{0}$ of $[A, t],[A] \neq \alpha, i \in \mathbf{d} \alpha,[\alpha i] \neq \tau i$

then

$$
[\alpha i, v \tau i] \in \mathbf{g} \mathscr{L}_{1} .
$$

Proof. Let $M$ be the set of all $[A, t]$ in $\mathbf{g} \mathscr{L}_{0}$ which satisfy (1). If $A \notin \mathscr{A}$ and $t \in \mathscr{L}_{0} A$ then $t \in \mathbb{I s}\{\psi[A, \alpha]\}$ for some $\alpha \in \mathscr{L} A$. Hence $v t=\alpha \in \mathscr{L} A=\mathscr{L}_{1} A$ and $[A, t] \in M$. 
Now we shall prove that if $[A, t]$ has a weakly $M$-regular structure $[\alpha, \tau]$ then both $(1)$ and (2) hold. If $[A]=\alpha$ then $[A, t] \in M$ and (2) is satisfied trivially. Let $[A] \neq \alpha$. Let $i \in \mathbf{d} \alpha,[\alpha i] \neq \tau i$. Then according to the assumption concerning $\mathscr{A}$ and $\omega$, we have $\alpha i \notin \mathscr{A}$. By (1) and weak $M$-regularity $v \tau i \in \mathbf{t}\left(\mathscr{L}_{1}, \alpha i\right)$. Hence (2) holds for our $A, t, \alpha$, $\tau$. We shall prove (1). Let $A \notin \mathscr{A}$. Then $v \alpha \in \mathscr{L}_{1} A$. If $[\alpha i] \neq \tau i$ then $(v \alpha) i=\alpha i$ and as we already know $v \tau i \in \mathbf{t}\left(\mathscr{L}_{1}, \alpha i\right)$. If $[\alpha i]=\tau i$ then $v[\alpha i]=(v \alpha) i=v \tau i$. Hence $\mathscr{L}_{1}: v \alpha \geqq \prod \mu \tau=v t$ and (1) holds, $[A, t] \in M$. According to Theorem 6.7, $M=\mathbf{g} \mathscr{L}$, every structure in $\mathscr{L}_{0}$ is weakly $M$-regular and the proof is complete.

9.11. Theorem. Let $\mathscr{L}$ be a language, $\mathscr{L}_{0}$ an $\omega$-isolation of $\mathscr{L}$. Then the structural unambiguity of $\mathscr{L}_{0}$ is sufficient and necessary for that of $\mathscr{L}$.

Proof. Let $\varrho$ be the reducing transformation satisfying (9.7.3). Suppose $\mathscr{L}$ is structurally ambiguous. Then there is a $\varrho$-invariant $g \in \mathbf{g} \mathscr{L}$ with two different structures (in $\mathscr{L})\left[\alpha_{1}, \tau_{1}\right],\left[\alpha_{2}, \tau_{2}\right]$ (see Theorem 9.4). According to Lemma 9.9, there is a grammatical element $g_{0} \in \mathbf{g} \mathscr{L}_{0}$ and two structures (in $\mathscr{L}_{0}$ ) $\left[\beta_{1}, \xi_{1}\right],\left[\beta_{2}, \xi_{2}\right]$ of $g_{0}$ such that $\alpha_{i}=v \beta_{i}, \tau_{i}=\mu \xi_{i}$ for $i=1$, 2. If $\mathscr{L}_{0}$ is structurally unambiguous then $\beta_{1}=\beta_{2}, \xi_{1}=\xi_{2}$, which implies $\alpha_{1}=\alpha_{2}, \tau_{1}=\tau_{2}$ contrary to the assumption. Hence $\mathscr{L}_{0}$ is structuraly ambiguous and the sufficiency is proved.

Now let $\mathscr{L}$ be structurally unambiguous and $\mathscr{L}_{0}$ structurally ambiguous, so that an $g_{0}=\left[A, t_{0}\right] \in \mathbf{g} \mathscr{L}_{0}$ has two different structures $\left[\beta_{1}, \xi_{1}\right],\left[\beta_{2}, \xi_{2}\right]$ in $\mathscr{L}_{0}$. Then by Lemma 9.8, $\left[v \beta_{i}, \mu \xi_{i}\right]$ are structures in $\mathscr{L}$ of an $g \in \mathbf{g} \mathscr{L}$ for $i=1,2$. Since $g$ has only one structure $[\alpha, \tau]$ in $\mathscr{L}$ we have $\alpha=v \beta_{i}, \tau=\mu \xi_{i}$. Because $\prod \xi_{1}=\prod \xi_{2}=t_{0}$ and $\mathbf{\iota} \xi_{i}=\mathbf{\imath} \tau$, we have $\xi_{1}=\xi_{2}$; set $\xi=\xi_{1}$ and observe that $\mathbf{d} \alpha=\mathbf{d} \beta_{1}=\mathbf{d} \beta_{2}=\mathbf{d} \xi$. Now for every $j \in \mathbf{d} \alpha$ and every $i \in\{0,1\}$ we have $\beta_{i} j \in\{\alpha j, \varphi \alpha j\}$. We shall prove $\beta_{1} j=\beta_{2} j$. If $\beta_{1} j \neq \beta_{2} j$ then we have, possibly after interchanging indices, $\beta_{1} j=\alpha j$, $\beta_{2} j=\varphi \alpha j \notin \mathbf{d} \mathscr{L}_{0}$. Hence in this case $\left[\beta_{2} j\right]=\xi j, \mathscr{L}_{0}:[\alpha j]=\left[\beta_{1} j\right] \rightarrow \xi j=$ $=\left[\beta_{2} j\right]=[\varphi \alpha j], \mathscr{L}_{0}:[\alpha j] \rightarrow[\varphi \alpha j] . \quad$ By Lemma $9.8 \quad \mathscr{L}:[\alpha j] \rightarrow[\alpha j]$, which contradicts the assumed structural unambiguity of $\mathscr{L}$ (see Theorem 7.6). Hence $\beta_{1} j=\beta_{2} j, \beta_{1}=\beta_{2}$ which contradicts the assumption, and the necessity is proved.

9.12. Theorem. Let $\mathscr{L}$ be a language, $\mathscr{L}_{1} \subset \mathscr{L}$, let $\mathscr{A}=\mathbf{d} \mathscr{L}-\mathbf{d} \mathscr{L}_{1}$ be disjoint with $\mathbf{a} \mathscr{L}_{1}$, let every $[A, t] \in \mathbf{g} \mathscr{L}$ be structurally unambiguous if $A \in \mathscr{A}$. Then the structural unambiguity of $\mathscr{L}_{1}$ is necessary and sufficient for that of $\mathscr{L}$.

Proof. The necessity follows from Lemma 8.3. Concerning sufficiency, let $\mathscr{L}_{1}$ be structurally unambiguous. Let $M=\{[A, t] ;[A, t] \in \mathbf{g} \mathscr{L}$ and (either $A \in \mathscr{A}$ or $\left.\left.[A, t] \in \mathbf{g} \mathscr{L}_{1}\right)\right\}$. If $t \in \mathscr{L} A$ then $[A, t] \in M$.

Let $g=[A, t]$ be in $\mathbf{g} \mathscr{L}$ and have a weakly $M$-regular structure $[\alpha, \tau]$ in $\mathscr{L}$, let $A \notin \mathscr{A}$. We shall show that $g \in \mathbf{g} \mathscr{L}_{1}$ and that $[\alpha, \tau]$ is a structure of $g$ in $\mathscr{L}_{1}$ also.

Suppose first $[A]=\alpha$. Then $t \in \mathscr{L} A=\mathscr{L}_{1} A$ and the assertion holds.

Secondly let $[A] \neq \alpha$. Then $\alpha \in \mathscr{L} A=\mathscr{L}_{1} A$ and if $[\alpha i] \neq \tau i$ then by weak $M$ regularity, $[\alpha i, \tau i] \in \mathbf{g} \mathscr{L}_{1}$ since by the assumption of the theorem, $\alpha i \notin \mathscr{A}$. Hence the assertion holds again. In particular $g \in M$, by Theorem $6.7 M=\mathbf{g} \mathscr{L}$, every structure 
in $\mathscr{L}$ is weakly $M$-regular. Now let $[A, t] \in \mathbf{g} \mathscr{L}$ have two structures $\left[\alpha_{1}, \tau_{1}\right],\left[\alpha_{2}, \tau_{2}\right]$ in $\mathscr{L}$. If $A \notin \mathscr{A}$ then by what we have already proved, $\left[\alpha_{i}, \tau_{i}\right]$ are structures of $[A, t]$ in $\mathscr{L}_{1}$, whence $\left[\alpha_{1}, \tau_{1}\right]=\left[\alpha_{2}, \tau_{2}\right]$. The same equality follows from the assumption of the theorem if $A \in \mathscr{A}$.

9.13. Theorem. Let $\mathscr{A}$ be an isolable subset of $\mathbf{d} \mathscr{L}$, let $\mathscr{L}_{1}=\mathscr{L}_{\mathbf{d} \mathscr{L}-\mathscr{A}}$ and let for every $A$ in $\mathscr{A}$ and $t$ in $\mathbf{t}(\mathscr{L}, A)$ there be at most one structure $[\alpha, \tau]$ in $\mathscr{L}$ of $[A, t]$ satisfying either

$$
[\alpha, \tau]=[[A],[t]]
$$

or

$$
[\alpha i, \tau i] \in \mathbf{g} \mathscr{L}_{1} \text { for every } i \in \mathbf{d} \alpha \text { such that }[\alpha i] \neq \tau i \text {. }
$$

Then the structural unambiguity of $\mathscr{L}_{1}$ is necessary and sufficient for that of $\mathscr{L}$.

Proof. The necessity follows from Lemma 8.3. Suppose $\mathscr{L}_{1}$ is structurally unambiguous. In view of Theorem 9.11 it suffices to prove that an isolation $\mathscr{L}_{0}$ of $\mathscr{L}$ is structurally unambiguous. Choose an isolating transformation $\omega$ in such a way that $\omega[A, \alpha, i] \neq \alpha i$ if and only if $\alpha i \in \mathscr{A}$; let $\mathscr{L}_{0}$ be the isolation of $\mathscr{L}$ by $\omega$. By (9.7.2.3), $\varphi a \neq a$ if and only if $a \in \mathscr{A}$ and by (9.7.6) and (9.7.7) this implies that the set Is $\{\psi[A, \alpha]\}$ contains exactly one element, namely $\psi[A, \alpha]$. We have $\psi[A, \alpha]=$ $=v_{0} \alpha, \mathscr{L}_{0} A=\left\{v_{0} \alpha ; \alpha \in A\right\}$.

Let $\left[\alpha_{1}, \tau_{1}\right],\left[\alpha_{2}, \tau_{2}\right]$ be two structures of $[A, t]$ in $\mathscr{L}_{0}$, let $A \in \mathscr{A}$. By Lemma 9.8, $\left[v \alpha_{1}, \mu \tau_{1}\right],\left[v \alpha_{2}, \mu \tau_{2}\right]$ are two structures in $\mathscr{L}$. From (9.10.2) we conclude for $i=1,2$ that $\left[v \alpha_{i}, \mu \tau_{i}\right]$ satisfies either (1) or (2). Hence $\left[v \alpha_{1}, \mu \tau_{1}\right]=\left[v \alpha_{2}, \mu \tau_{2}\right]$. Since $\alpha_{i}=$ $=v_{0} v \alpha_{i}, \tau_{i}=\mu_{0} \mu \tau_{i}$, we have $\left[\alpha_{1} \tau_{1}\right]=\left[\alpha_{2}, \tau_{2}\right]$ and every $[A, t] \in \mathbf{g} \mathscr{L}_{0}$ with $A \in \mathscr{A}$ is structurally unambiguous.

Set since $\mathscr{A} \cap \mathbf{a} \widehat{\mathscr{L}}=\Lambda$ we conclude from Theorem 9.12 that $\mathscr{L}_{0}$ is structurally unambiguous if $\widehat{\mathscr{L}}=\left(\mathscr{L}_{0}\right)_{\mathrm{d} \mathscr{L}_{1}}$ has this property. We have assumed that $\mathscr{L}_{1}$ is structurally unambiguous and $\hat{\mathscr{L}}$ differs from $\mathscr{L}_{1}$ in that terminal symbols $a$ in $\mathscr{A}$ of $\mathscr{L}_{1}$ are replaced in $\widehat{\mathscr{L}}$ by terminal symbols $\varphi a$. Since $\varphi$ is one-to-one, the structural unambiguity of $\mathscr{L}_{1}$ implies that of $\widehat{\mathscr{L}}$, which was to be proved.

\section{ISOLABLE SYMBOLS}

10.1. Remark. In this section we shall formulate some assymmetrical conditions which are sufficient for the existence of isolating transformations. Roughly speaking, they correspond to the case in which it is easier to recognize the end of an inserted text than its beginning. It is possible to formulate and prove the results corresponding to the other case. For this it sufficies to consider another language $\mathscr{L}_{1}$ with $\mathbf{d} \mathscr{L}=\mathbf{d} \mathscr{L}_{1}$ and $\mathscr{L}_{1} A=\left\{\beta ; \beta_{i}=\alpha(\lambda \alpha+1-i), i \in \mathbf{d} \alpha, \lambda \alpha=\lambda \beta, \alpha \in \mathscr{L} A\right\}$. 
10.2. Definition. A subset $\mathscr{A}$ of $\mathbf{d} \mathscr{L}$ is said to be complete if for every $t$ in $\bigcup\{\mathbf{t}(\mathscr{L}, A) ; A \in \mathscr{A}\}$ there is an $A_{0} \in \mathscr{A}$ such that

$$
[B] \rightarrow t, \quad B \in \mathbf{d} \mathscr{L}
$$

implies

$$
\left.[B] \rightrightarrows\left[A_{0}\right], \quad \boldsymbol{\delta}_{0}\left[B,\left[A_{0}\right]\right]<\boldsymbol{\delta}_{0}[B, t], \quad\left[A_{0}\right] \rightarrow\left[A_{0}\right] \rightarrow t .^{1}\right)
$$

10.3. Definition. Let $\mathscr{A} \subset \mathbf{d} \mathscr{L}$. We say that $\mathscr{A}$ is $f$-recognizable if $f$ is a transformation defined on $\mathbf{g} \mathscr{L}, \mathbf{g}[A, t] \subset \mathbf{d} t$ and the following two conditions hold for every $[A, t] \in$ if $f \mathscr{L}$ :

$$
f[A, t] \neq \Lambda \text { if } A \in \mathscr{A} ; f[A, t]=\Lambda \text { if } A \notin \mathscr{A}, t \in \mathscr{L} A .
$$

(2) If $g=[A, t] \in \mathbf{g} \mathscr{L}$ and $[\alpha, \tau]$ is a structure of $g, x=\mathbf{\imath} \tau, \alpha \neq[A]$, then

$$
\begin{gathered}
f g=\{i ; i=x j, j \in f[A, \alpha],[\alpha j]=\tau j\} \cup \\
\cup\{i ; i=x j-1+s, j \in \mathbf{d} \alpha,[\alpha j] \neq \tau j, s \in f[\alpha j, \tau j]\} .
\end{gathered}
$$

10.4. Lemma. Let $\mathscr{A} \subset \mathbf{d} \mathscr{L}, Q \subset \mathbf{a}_{\mathrm{t}} \mathscr{L}$, for every $\alpha \in \cup \mathbf{r} \mathscr{L}$ let $Q$ be disjoint with symb $\{\alpha\}$ if and only if $\alpha \notin \bigcup\{\mathscr{L} A ; A \in \mathscr{A}\}$.

Then there is an $f$ 'such that $\mathscr{A}$ is $f$-recognizable.

Proof. Put $f[A, t]=\{i ; i \in \mathbf{d} t, t i \in Q\}$ for every $[A, t] \in \mathbf{g} \mathscr{L}$.

10.5. Definition. Let $\mathscr{A}$ be an $f$-recognizable subset of $\mathbf{d} \mathscr{L}$. We say that $f_{0}$ and $f_{1}$ indicate the beginning and the end for $f$, if $f_{0}, f_{1}$ are functions defined on the set $\{[A, t, i] ;[A, t] \in \mathbf{g} \mathscr{L}, i \in f[A, t]\}$ with values $f_{j}[A, t, i] \in \mathbf{d} t, f_{0}[A, t, i] \leqq i \leqq$ $\leqq f_{1}[A, t, i]$ and if the following conditions are satisfied for every $g=[A, t] \in \mathbf{g} \mathscr{L}$ and each of its structures $[\alpha, \tau], i \in f g, x=\mathbf{\imath} \tau, x j \leqq i<x(j+1)$ :

(1) if $[A] \Rightarrow t$ then $f_{0}[A, t, i]=1, f_{1}[A, t, i]=\lambda t$,

(2) if $[\alpha j]=\tau j$ and if for every $i_{0}<i, i_{0} \in f g$ we have $f_{0}\left[A, t, i_{0}\right]=1$,

$f_{1}\left[A, t, i_{0}\right]=\lambda t$

then

$$
f_{0}[A, t, i]=1, f_{1}[A, t, i]=\lambda t,
$$

(3) if $[\alpha j] \neq \tau j$ then for $s=0,1$

$$
f_{s}[A, t, i]=x j-1+f_{s}[\alpha j, \tau j, i+1-x j] .
$$

(We note that the right-hand side of the equality in (3) is meaningful according to (10.3.2).)

1) We writte " $a \nrightarrow b$ " for "non $(a \rightarrow b)$ ". 
10.6. Definition. Let $\mathscr{A} \subset \mathbf{d} \mathscr{L}$. We define the sets of left and right delimiters for $\mathscr{A}$ in the following way:

(1) Idel $\mathscr{A}=\operatorname{symb}_{\mathrm{e}}\left\{t ; \alpha \in \bigcup \mathbf{r} \mathscr{L}, i \in \mathbf{d} \alpha,[\alpha i] \rightrightarrows \beta, \beta 1 \in \mathscr{A}, \alpha^{(1, i-1)} \rightrightarrows t\right\}$,

(2) $\mathbf{r d e l} \mathscr{A}=\operatorname{symb}_{\mathrm{b}}\left\{t ; \alpha \in \bigcup \mathbf{r} \mathscr{L}, i \in \mathbf{d} \alpha,[\alpha i] \rightrightarrows \beta, \beta \lambda \beta \in \mathscr{A}, \alpha^{(i+1, \lambda \alpha)} \rightrightarrows t\right\}$.

10.7. Lemma. Let $\mathscr{A} \subset \mathbf{d} \mathscr{L}$ be $f$-recognizable, let $k_{0}$ and $k_{1}$ be two integer-valued functions defined on

$$
Q=\{t i ; i \in f[A, t], A \Rightarrow t\} .
$$

Let $Q \subset \mathbf{a}_{\mathbf{t}} \mathscr{L}$, for every $q \in Q$ let $\mathscr{B}_{q}=\{\alpha ;[A] \Rightarrow \alpha, i \in f[A, \alpha], \alpha i=q\}, \mathscr{A}_{q}=$ $=\left\{A ;[A] \Rightarrow \alpha \in \mathscr{B}_{q}\right\}, \mathscr{T}_{q}=\left\{t ; \beta \rightrightarrows t, \beta \in \mathscr{B}_{q}\right\}$.

For each $q$ in $Q$ let one of the conditions (2a), (2b), (2c) and also one of (3a), (3b), (3c) hold for every $A, \alpha, j$ such that

$$
[A] \Rightarrow \alpha, j \in f[A, \alpha], \alpha j=q:
$$

$$
\text { if } \alpha^{(j+1, \lambda \alpha)} \rightrightarrows u \text { then } \lambda u=k_{1} q \text {, }
$$

$$
\begin{gathered}
\left(\text { symb }_{\mathrm{e}} \mathscr{T}_{q}\right) \cap\left(\text { symb }\left\{u^{(1, \lambda u-1)} ; \alpha^{(j, \lambda \alpha)} \rightrightarrows u\right\}\right)=\Lambda, \\
\left(\text { rdel } \mathscr{A}_{q}\right) \cap\left(\text { symb }\left\{u ; \alpha^{(j+1, \lambda \alpha)} \rightrightarrows u\right\}\right)=\Lambda, \\
\text { if } \alpha^{(1, j-1)} \rightrightarrows u \text { then } \lambda u=k_{0} q, \\
\left(\text { symb }_{\mathrm{b}} \mathscr{T}_{q}\right) \cap\left(\operatorname { s y m b } \left(\left\{\alpha^{(2, j)}\right\} \cup\right.\right. \\
\cup\{u ; 1<h<j,[\alpha h] \rightarrow u, f[\alpha h, u]=\Lambda\} \cup \\
\left.\left.\cup\left\{u^{(2, \lambda u)} ;[\alpha 1] \rightarrow u, f[\alpha 1, u]=\Lambda\right\}\right)\right)=\Lambda,
\end{gathered}
$$

$$
\begin{gathered}
\left(\text { Idel } \mathscr{A}_{q}\right) \cap\left(\operatorname { s y m b } \left(\left\{\alpha^{(1, j-1)}\right\} \cup\right.\right. \\
\cup\{u ; 1 \leqq h<j,[\alpha h] \rightarrow u, f[\alpha h, u]=\Lambda\}))=\Lambda .
\end{gathered}
$$

Then there are $f_{0}$ and $f_{1}$ which indicate the beginning and the end for $f$.

Proof. From (10.3.2), $Q=\{t i ; A \in \mathbf{d} \mathscr{L},[A] \rightarrow t, i \in f[A, t]\}$. Hence if $[A, t] \in$ $\in \mathbf{g} \mathscr{L}, i \in f[A, t], q=t i$ then $q \in Q$. Set $u=[A, t, i]$ and define $f_{0} u$ and $f_{1} u$ as the largest and smallest integers respectively, for which $f_{0} u \leqq i \leqq f_{1} u$ and either $f_{1} u=$ $=\lambda t$ or

(4) if (1) implies (2a) then

$$
f_{1} u=i+k_{1} q
$$

else if (1) implies (2b) then

$$
t f_{1} u \in \operatorname{symb}_{\mathrm{e}} \mathscr{T}_{q}
$$

else

$$
t\left(f_{1} u+1\right) \in \operatorname{rdel} \mathscr{A}_{q},
$$


and either $f_{0} u=1$ or

(5) if (1) implies (3a) then

$$
f_{0} u=i-k_{0} q
$$

else if (1) implies (3b) then

$$
t f_{0} u \in \mathbf{s y m b}_{\mathrm{b}} \mathscr{T}_{q}
$$

else

$$
t\left(f_{0} u-1\right) \in \text { Idel } \mathscr{A}_{q} .
$$

Now let us state two assumptions:

(6a)

$$
g=[A, t] \in \mathbf{g} \mathscr{L}, i \in f g, q=t i,
$$

(6b) condition (6a) holds, $[A] \Rightarrow \alpha, \tau$ is an $\alpha$-decomposition of $t, x=\mathbf{\imath} \tau$, $x j \leqq i<x(j+1), p=x j-1$.

Directly from the definition of $f_{0}$ and $f_{1}$ we conclude:

(7a) if $(6 b)$ holds and $[\alpha j] \neq \tau j$ then

$f_{0}[\alpha j, \tau j, i-p]>1$ implies $f_{0}[A, t, i]=f_{0}[\alpha j, \tau j, i-p]+p ;$

$f_{0}[\alpha j, \tau j, i-p]=1$ implies $f_{0}[A, t, i] \leqq p+1$

and

(7b) if $(6 \mathrm{~b})$ holds and $[\alpha j] \neq \tau j$ then

$f_{1}[\alpha j, \tau j, i-p]<\lambda \tau j$ implies $f_{1}[A, t, i]=f_{1}[\alpha j, \tau j, i-p]+p ;$

$f_{1}[\alpha \mathrm{j}, \tau \mathrm{j}, i-p]=\lambda \tau \mathrm{j}$-implies $f_{1}[A, t, i] \geqq x(j+1)-1$.

We shall prove the following three properties:

(8a) if (6a) holds and $f_{0}[A, t, i]=1$ then there is a $\beta$ such that $[A] \rightrightarrows$

$\Longrightarrow \beta \equiv t, \beta 1 \in \mathscr{A}_{q}, t 1 \in \mathbf{s y m b}_{\mathbf{b}} \mathscr{T}_{q}$, and $k_{0} q=i-1$ if (1) implies (3a)

(8b) if (6a) holds and $f_{1}[A, t, i]=\lambda t$ then there is a $\beta$ such that $[A] \rightrightarrows$

$\rightrightarrows \beta \equiv t, \beta \lambda \beta \in \mathscr{A}_{q}, t \lambda t \in \mathbf{s y m b}_{\mathrm{e}} \mathscr{T}_{q}$, and $k_{1} q=\lambda t-i$ (1) implies (2a)

(9) if (6b) holds and $[\alpha j] \neq \tau j$ then $x j \leqq f_{0}[A, t, i]$ and $f_{1}[A, t, i]<x(j+1)$.

Let $M$ be the set of all grammatical elements $g=[A, t]$ for which (8a) and (8b) hold. If $[A] \Rightarrow t$ then $A \in \mathscr{A}_{q}$ and both (8a) and (8b) hold.

Now if (6a) holds and $[\alpha, \tau]$ is a structure of $[A, t]$ then (6b) holds with suitably chosen $x, j, p$. Hence in proving (8a) and (8b) we may assume that not only (6a) but 
also (6b) holds. Moreover, assume $[\alpha h, \tau h] \in M$ whenever $[\alpha h] \neq \tau h$, and prove that then (8a), (8b) and (9) hold. According to Theorem 6.7 we shall then have $M=\mathbf{g} \mathscr{L}$, the additional condition will be a consequence of $(6 \mathrm{~b})$ and, hence, the proof of $(8 \mathrm{a})$, $(8 b)$ and $(9)$ will be completed.

First let $[\alpha j] \neq \tau j$. If $f_{0}[\alpha j, \tau j, i-p]>1$ then $f_{0}[A, t, i]>x j$ according to $(7 \mathrm{a})$. Hence in this case (8a) and the first inequality in (9) hold. Let $f_{0}[\alpha j, \tau j, i-p]=1$. Since $[\alpha j, \tau j] \in M$, there is a $\beta_{0}$ such that $[\alpha j] \rightrightarrows \beta_{0} \supseteqq \tau j, \beta_{0} 1 \in \mathscr{A}_{q}, \tau j 1 \in \mathbf{s y m b}_{\mathrm{b}} \mathscr{T}_{q}$. If $x j=1$ then (8a) holds with $\beta=\beta_{0} \times \prod \tau^{(j+1, \lambda \tau)}$ and the first inequality in (9) also. holds. Let $x j>1$. The first equation in (9) obviously holds if, for our $q$, (1) implies (3a) or (1) implies (3b). Otherwise (1) implies (3c) and from $\beta_{0} \rightrightarrows \tau j, \beta_{0} 1 \in \mathscr{A}_{q}$ we get $t(x j-1) \in \mathbf{I d e l} \mathscr{A}_{q}$, and, consequently by $(5 \mathrm{c}) f[A, t, i] \geqq x j>1$, the first inequality in (9) holds again and (8a) is satisfied trivially. (8b) and the second inequality in (9) can be proved similarly.

Secondly, let $[\alpha j]=\tau j$. Then (9) is satisfied trivially and (8a) and (8b) hold with $\beta=[A]$ because $j \in f[A, \alpha]$ by (10.3.2), $\alpha j=q, \alpha \in \mathscr{B}_{q}, \beta 1=\beta \lambda \beta=A \in \mathscr{A}_{q}$. This completes the proof of (8a), (8b) and (9). Combining (9), (7a) and (7b) we obtain

(10) if $(6 \mathrm{~b})$ holds and $[\alpha j] \neq \tau j$ then for $s=0,1$

$$
f_{s}[A, t, i]=f_{s}[\alpha j, \tau j, i-p]+p .
$$

As the next step we shall prove:

(11) if

(11a) condition (6b) holds, and for every $i_{0}<i, i_{0} \in f g$ we have

$$
f_{0}\left[A, t, i_{0}\right]=1, f_{1}\left[A, t, i_{0}\right]=\lambda t,
$$

then

$$
\bigcup\{f[\alpha h, \tau h] ; \quad 1 \leqq h<j, \quad[\alpha h] \neq \tau h\}=\Lambda
$$

and

(11c) $[\alpha j]=\tau j$ implies $f_{0}[A, t, i]=1, f_{1}[A, t, i]=\lambda t$.

Let (11a) hold but let there be an $h<j$ such that $[\alpha h] \neq \tau h, i_{0} \in f[\alpha h, \tau h]$ for some $i_{0}$. By (11a) $f_{0}\left[A, t, i_{0}\right]=1, f_{1}\left[A, t, i_{0}\right]=\lambda t$. According to (9), this implies $\lambda t<$ $<x(h+1) \leqq x j$ which contradicts the assumption $i \in f[A, t], i \geqq x j$. Hence $(11 b)$. holds. We shall prove (11c). Let $[\alpha j]=\tau j$. We are to prove $f_{0}[A, t, i]=1$, $f_{1}[A, t, i]=\lambda t$. We shall prove only the first equality; the proof of the second equality is analoguous and a little simpler. We may assume $i>1$ since otherwise $1 \leqq f_{0}[A, t, i] \leqq i=1$. First let, for our $q$,(1)imply (3a). Then $\alpha^{(1, j-1)} \geqq \prod \tau^{(1, j-1)}$, $k_{0} q=\lambda \prod \tau^{(1, j-1)}=x j-1=i-1$ and by $(5 \mathrm{a}) f_{0}[A, t, i]=i-(i-1)=1$. Secondly, let (1) not imply (3a). Denote by $R_{\mathrm{b}}$ and $R_{\mathrm{c}}$ the right-hand terms of the intersections in (3b) and (3c), respectively. If, for an $k<j,[\alpha h] \neq \tau h$ then by (11b), 
$f[\alpha h, \tau h]=\Lambda$. Hence $R_{\mathrm{b}} \supset \operatorname{symb}\left(\left\{\alpha^{(2, j)}\right\} \cup\{\tau h ; 1<h<j\} \cup\left\{(\tau 1)^{(2, \lambda \tau 1)}\right\}\right) \supset$ $\supset$ symb $\left\{t^{(2, i)}\right\}$. Similarly $R_{\mathrm{c}} \supset$ symb $\left\{t^{(1, i-1)}\right\}$. If $(1)$ implies $(3 \mathrm{~b})$ then $\mathbf{s y m b}_{\mathrm{b}} \mathscr{T}_{\mathrm{q}} \cap$ $\cap \operatorname{symb}\left\{t^{(2, i)}\right\}=\Lambda$ and $f_{0}[A, t, i]=1$. Otherwise (1) implies (3c), Idel $\mathscr{A}_{q} \cap$ symb $\left\{t^{(1, i-1)}\right\}=\Lambda$ and by $(5 \mathrm{c}), f_{0}[A, t, i]=1$ again; the proof of (11) is completed.

Now we see that all the conditions of Definition 10.5 are satisfied: (10.5.1) follows from (11) applied for $\alpha=t, \tau=\boldsymbol{\delta}_{\mathrm{p}} t$ successively for all $i \in f[A, t]$. (10.5.2) follows from (11) also and (10) implies (10.5.3).

10.8. Theorem. Let $\mathscr{A}$ be a complete and f-recongnizable subset of $\mathbf{d} \mathscr{L}$, let $f_{0}$ and $f_{1}$ indicate the beginning and the end for $f$, let $\omega$ and $\varphi$ satisfy conditions (9.7.1)-(9.7.2), let $\omega[A, \alpha, i]=\alpha i$ unless $\alpha i \in \mathscr{A}$ and either

$$
\text { not } \alpha^{(1, i-1)} \times \alpha^{(i+1, \lambda \alpha)} \rightrightarrows \Lambda
$$

or

(2) the condition that

$$
[\alpha i] \rightarrow t,[B] \rightrightarrows[A],[\beta, \tau] \text { is a structure of }[B, t]
$$

implies

(2b) there is an $s \in \mathbf{d} \tau$ such that $[\beta s] \rightarrow \tau s=t$.

Then $\omega$ is an isolating transformation.

Proof. For every $g=[A, t] \in \mathbf{g} \mathscr{L}$ denote by $\mathscr{I}_{0} g$ the subset of $f g$ containing all such $i$ for which $f_{0}[A, t, i]=1, f_{1}[A, t, i]=\lambda t$. Let $\mathscr{I} g$ be the set containing the smallest element of the set $f g-\mathscr{I}_{0} g$, if this set is non-empty; otherwise put $\mathscr{I} g=\Lambda$.

First we shall prove the following assertion:

$$
\text { If }[A, t] \in \mathbf{g} \mathscr{L} \text { and } \mathscr{I}_{0} g \neq \Lambda \text { then } t \in \bigcup\{\mathbf{t}(\mathscr{L}, A) ; A \in \mathscr{A}\} \text {. }
$$

Let $M$ be the set of all such $[A, t] \in \mathbf{g} \mathscr{L}$ for which (3) holds. Suppose $[A] \Rightarrow t$. Then $\mathscr{I}_{0} g \neq \Lambda$ implies $f g \neq \Lambda$ and according to (10.3.1) this implies that $A \in \mathscr{A}$; hence $[A, t] \in M$. Now suppose that $[A, t]$ has a weakly $M$-regular structure $[\alpha, \tau]$. Choose $i \in \mathscr{I}_{0}(g)$, let $j$ be such that $x j \leqq i<x(j+1)$, where $x=\mathbf{\imath} \tau$. If $[\alpha j]=\tau j$ then by Definition 10.3, $A \in \mathscr{A}$. If $[\alpha j] \neq \tau j$ then by $(10.5 .3) \tau j=t, \mathscr{I}_{0}[\alpha j, \tau j] \neq \Lambda$ and since we assume $[\alpha j, \tau j] \in M$, there is an $A_{0} \in \mathscr{A}$ for which $\left[A_{0}\right] \rightarrow \tau j=t$. According to Theorem 6.7, this completes the proof of (3). By the completeness of $\mathscr{A}$ (see Definition 10.2) and by (3) there is a transformation $v$ defined on $\delta \mathscr{L}$ such that (4) $[v t] \neq t$, and if

$$
[A, t] \in \mathbf{g} \mathscr{L}, \quad \mathscr{I}_{0}[A, t] \neq \Lambda, \quad B \in \mathbf{d} \mathscr{L}, \quad[B] \rightarrow t
$$

then

$$
[B] \rightrightarrows[v t] \rightarrow t, \quad \delta_{0}[B,[v t]]<\delta_{0}[B, t], \quad[v t] \rightarrow[v t]
$$


In the next step we shall define two transformations $V, R$ on $\mathbf{g} \mathscr{L}$ in the following manner:

If $g=[A, t] \in \mathbf{g} \mathscr{L}$ and if

$$
\mathscr{I} g \neq \Lambda
$$

then

$$
\begin{gathered}
V g=t^{\left(1, i_{0}-1\right)} \times\left[v t^{\left(i_{0}, i_{1}\right)}\right] \times t^{\left(i_{1}+1, \lambda t\right)}, \\
R g=\delta_{\mathrm{p}} t^{\left(1, i_{0}-1\right)} \times\left[t^{\left(i_{0}, i_{1}\right)}\right] \times \delta_{\mathrm{p}} t^{\left(i_{1}+1, \lambda t\right)},
\end{gathered}
$$

where

$$
s \in \mathscr{I} g, \quad i_{0}=f_{0}[A, t, s], \quad i_{1}=f_{1}[A, t, s]
$$

if

(6a) $\mathscr{I} g=\Lambda, f g \neq \Lambda, v t \neq[A]$ and $(2 \mathrm{~b})$ holds for every structure $[\beta, \tau]$ of a grammatical element $[B, t]$ with $[B] \geqq[A]$,

then

$$
V g=[v t], \quad R g=[t]
$$

if

(7a) neither (5a) nor (6a) holds

then

$$
V g=t, \quad R g=\delta_{p} t
$$

We shall show that $\langle V, R\rangle$ is a reducing pair. Let $g=[A, t] \in \mathbf{g} \mathscr{L}$, let $\varrho$ be defined as in Definition 9.1. By the definition of $\mathscr{I} g$ we have $\lambda V g>1$ if (5a) holds; $V g=$ $=[v t] \neq[A]$ if $(6 \mathrm{a})$ holds; otherwise by $(7 \mathrm{~b}), V g=t$. Hence the relation $t \neq V g=$ $=[A]$ cannot hold and (9.1.2) is satisfied.

For the proof of (9.1.1), (9.1.3), (9.1.4) and (9.1.5) we shall use Theorem 9.6. Clearly, condition (9.6.1) is satisfied. If $[\alpha, \tau]$ is a structure of $g$ then $R g$ is finer than $\tau$. This is obvious if (7a) or (6a) holds, and it is a consequence of $(10.5 .1)-(10.5 .3)$ if (5a) holds. Hence there is an index-decomposition $x_{0}$ of $R g$ (and of $V g$ also, because $\lambda V g=\lambda R g)$ such that the decomposition $\zeta=\delta\left(R g, x_{0}\right)$ satisfies (9.6.2a). Because $(R g) i \neq \Lambda$ for every $i \in \mathbf{d} R g$, we obtain from Lemma 4.11 that $x_{0}$ is determined uniquely. We shall prove that $\zeta$ and $\xi=\delta\left(V g, x_{0}\right)$ satisfy, for every $i \in \mathbf{d} \alpha$, at least one of conditions (9.6.2b1), (9.6.2b2), (9.6.2b3); this is sufficient to prove (9.6.2).

Put $x=\mathrm{\imath} \tau, x_{1}=\mathbf{\imath} R$.

First let (7b) hold. Then $x_{1}$ is the identical transformation on $I(\lambda t+1), x_{0}=x$, $\xi=\delta\left(V g, x_{0}\right)=\delta(t, x)=\tau, \quad \zeta=\delta\left(\delta_{\mathrm{p}} t, x\right), \quad \xi i=\tau i, \quad \zeta i=\left(\boldsymbol{\delta}_{\mathrm{p}} t\right)^{(x i, x(i+1)-1)}=\boldsymbol{\delta}_{\mathrm{p}} \tau i$ and (9.6.2b3) holds. 
Secondly let (6a) hold. Then there is an $s$ such that $[\alpha s] \rightarrow t=\tau s$ hence $\lambda \xi i=\lambda \zeta i$ is equal to zero and one for $i \neq s$ and $i=s$, respectively. For $i \neq s, i \in \mathbf{d} \alpha, \xi i=$ $=\zeta i=\Lambda$ satisfy (9.6.2b3). For $i=s$ we obtain from (6b) $\xi i=[v t]$ and from (4) $[\alpha i] \rightrightarrows[v t]=\xi i \rightarrow t=\tau i, \delta_{0}[\alpha i, \xi i]<\delta_{0}[\alpha i, \tau i]$ and (9.6.2b1) holds.

Thirdly let (5a) hold. Let $s \in \mathscr{I} g, x j \leqq s<x(j+1)$. By (10.5.2) we have $[\alpha j] \neq \tau j$. If $i \neq j$ then according to $(5 b), \zeta i=\delta_{\mathrm{p}} t^{(x i, x(i+1)-1)}=\delta_{\mathrm{p}} \tau i, \xi i=t^{(x i, x(i+1)-1)}=\tau i$ and (9.6.2b3) holds.

If $i=j$ then

$$
\begin{gathered}
\zeta i=\left(\delta_{\mathrm{p}} t^{\left(x i, i_{0}-1\right)}\right) \times\left[t^{\left(i_{0}, i_{1}\right)}\right] \times\left(\delta_{\mathrm{p}} t^{\left(i_{1}+1, x(i+1)-1\right)}\right), \\
\xi i=t^{\left(x i, i_{0}-1\right)} \times\left(v t^{\left(i_{0}, i_{1}\right)}\right) \times t^{\left(i_{1}, x(i+1)-1\right)}
\end{gathered}
$$

with $i_{0}, i_{1}$ as in $(5 b)$. First let $\left[i_{0}, i_{1}\right] \neq[x i, x(i+1)]$. Then according to (10.5.3), $\mathscr{I}[\alpha i, \tau i]=\{s+x i-1\}$. Hence in this case $\xi i=V[\alpha i, \tau i], \zeta i=R[\alpha i, \tau i]$. According to (10.5.1), from $\mathscr{I} g \neq \Lambda$ we obtain further that $t \notin \mathscr{L} A$ and hence $\alpha \neq[A]$. Thus in this case $(9.6 .2 \mathrm{~b} 2)$ holds. Secondly let $\left[i_{0}, i_{1}\right]=[x i, x(i+1)]$. Then $\xi i=$ $=[v \tau i], \mathscr{I}_{0}[\alpha i, \tau i] \neq \Lambda$ and from (4) $[\alpha i] \rightrightarrows \xi i \rightarrow \tau i, \lambda \xi i=1, \delta_{0}[\alpha i, \xi i]<\delta_{0}[\alpha i, \tau i]$ and (9.6.2b1) holds.

This concludes the proof of condition (9.6.2). According to Theorem 9.6, if also (9.1.6) holds then $\langle V, R\rangle$ is a reducing pair. Let us prove (9.1.6). First note that $\varrho g=g$ if and only if (7a) holds; this follows from the property $[v t] \neq t$ for every $t \in \mathbf{d} v$. Now suppose that $\varrho[\alpha i, \tau i] \neq[\alpha i, \tau i]$ for some $i \in \mathbf{d} \alpha,[\alpha i] \neq \tau i$. If $\mathscr{I}[\alpha i, \tau i] \neq \Lambda$, then $\mathscr{I} g \neq \Lambda$ and $\varrho g \neq g$ according to $(5 \mathrm{a})$ and $(5 \mathrm{~b})$. Otherwise [ $[\alpha i, \tau i]$ must satisfy condition (6a) (for $[\alpha i, \tau i]$ substituted for $[A, t]$ ). In this case $f g \neq \Lambda, v \tau i \neq[\alpha i]$. If $\mathscr{I} g \neq \Lambda$ then again $\varrho g \neq g$ by $(5 \mathrm{a})$ and (5b). If $\mathscr{I} g=\Lambda$ then according to (10.5.3), $[\alpha i, \tau i]=[\alpha i, t]$. Since $[A] \rightrightarrows[\alpha i] \rightarrow[v t] \rightarrow[v t]$, we have $A \neq v t$. The remainder of condition (6a) is clearly satisfied for $g$ as well as for $[\alpha i, t]$. Hence (6a) holds for $g$ and $\varrho g \neq g$ again. This completes the proof of $(9.1 .6))$, which is the last condition needed to verify that $\langle V, R\rangle$ is a reducing pair.

For the proof of the theorem we must now show that $\langle V, R\rangle$ and $\varrho$ have the property (9.7.3). Let $A, t, \alpha, \tau, i$ be as in that condition, let $\omega[A, \alpha, i] \neq \alpha i,[\alpha i] \neq \tau i$. We have $[A] \Rightarrow \alpha$ and proceed to show that $g$ satisfies either (5a) or (6a), i.e., that $\varrho g \neq g$. According to the assumptions of the theorem, $\omega[A, \alpha, i] \neq \alpha i$ implies that $\alpha i \in \mathscr{A}$ and either (1) or (2) holds. $\alpha i \in \mathscr{A}$ implies $f[\alpha i, \tau i] \neq \Lambda$ and, by (10.3.1), $f g \neq \Lambda$. If $\mathscr{I} g \neq \Lambda$ then (5a) holds and $\varrho g \neq g$, and this is the case if (1) holds. Hence it remains to consider the case in which (2) holds, (1) does not hold and $\mathscr{I} g=\Lambda$. Then $\alpha^{(1, i-1)} \times \alpha^{(i+1, \lambda \alpha)} \rightrightarrows \Lambda,[\alpha i] \rightrightarrows \alpha \rightarrow t$ and by $(4),[A] \rightarrow[\alpha i] \rightrightarrows[v t] \rightarrow[v t]$ which implies $[A] \neq v t$. Since $[\alpha i] \rightarrow \tau i=t$, condition (2) implies (6a) and again $\varrho g \neq g$, which completes the proof.

10.9. Example. Let $\Delta_{1}, \Delta_{2}, \ldots, \Delta_{m},\left\{e_{0}\right\}, E=\left\{e_{1}, e_{2}, \ldots, e_{n}\right\}$ be disjoint sets, let $\mathscr{L}$ be a language with $\mathbf{d} \mathscr{L}=E$ and, for every $i \in I(n)$, let

$$
\mathscr{L} e_{i} \subset \bigcup\left\{\left\{e_{i-1}, \delta_{i}, e_{i} \delta_{i}, \delta_{i} e_{i-1}, e_{i} \delta_{i} e_{i-1}\right\} ; \delta_{i} \in \Delta_{i}\right\} .
$$


For example, $\mathscr{L} e_{1}$ may be equal to $\bigcup\left\{\left\{\delta_{1}, e_{1} \delta_{1}\right\} ; \delta_{1} \in \Delta_{1}\right\}$. The terminal $e_{1}$-texts are then in $\mathbf{s} \Delta_{1}$ and may be called primaries. Elements of $\Delta_{i}(i>1)$ may be called operators with priority $i$ and texts derived from $e_{i}$ may be called expressions of order $i$.

Put $\mathscr{A}=\left\{e_{1}\right\}$. Let $e_{i} \rightarrow t, e_{1} \rightarrow t$. Then $\{t\} \subset\left\{e_{0}\right\} \cup \Delta_{1}$, and every derivation $\sigma$ of $t$ from $e_{i}$ has a part $\sigma^{(1, i)}=\left[e_{i}, e_{i-1}, e_{i-2}, \ldots, e_{1}\right]$. Hence $e_{i} \rightrightarrows e_{1}, \delta_{0}\left[e_{i}, e_{1}\right] \leqq$ $\leqq \delta_{0}\left[e_{i}, t\right], e_{1} \rightarrow e_{1}$ and $\mathscr{A}$ is complete by Definition 10.2. Now let $Q_{0}=\Delta_{1} \cup\left\{e_{0}\right\}$ and $f[A, t]=\left\{i ; t i \in Q_{0}\right\}$ By Lemma $10.4 \mathscr{A}$ is $f$-recognizable. We shall proceed to show that the assumptions of Lemma 10.7 are satisfied and that, in particular, (10.7.1) implies (10.7.2c) and (10.7.3c). We have $Q \subset\left\{e_{0}\right\} \cup \Delta_{1}$, and $\mathscr{A}_{q}=\left\{e_{1}\right\}$ for every $q \in Q$. If $\alpha, \beta, t$ are as in (10.6.1) and if $t \neq \Lambda$ then $\beta 1=e_{1}, \alpha i \in E$, $i>1, \alpha(i-1) \in \Delta_{j}, j>1$ and hence Idel $\mathscr{A}_{q} \subset \bigcup_{j=2}^{n} \Delta_{j}$. If $\alpha, \beta, t$ are as in (10.6.2) then again $\alpha i \in E$ but $i<\lambda \alpha \geqq 2$, hence $\alpha^{(i+1)} \in \Delta_{j}, j \geqq 1$ and rdel $\mathscr{A}_{q} \subset$ $\subset \bigcup_{j=1}^{n} \Delta_{j}$. Now let (10.7.1) hold. Then $A=e_{1}, \alpha \in\left\{e_{0}, \delta_{1}, e_{1} \delta_{1}, \delta_{1} e_{0}, e_{1} \delta_{1} e_{0}\right\}$ with $\delta_{1} \in \Delta_{1}$ and $\alpha j \in Q$. Hence $\alpha^{(j+1, \lambda \alpha)}$ is either empty or equal to $e_{0}$, and the right-hand side of the intersection in $(10.7 .2 \mathrm{c})$ is part of the set $\left\{e_{0}\right\}$ which is disjoint with rdel $\mathscr{A}_{q}$. Hence condition (10.7.2c) holds. Concerning (10.7.3c), $\alpha^{(1, j-1)}$ is either empty or equal to $e_{1}$ or $\delta_{1}$ or $e_{1} \delta_{1}$, so that $(10.7 .3 \mathrm{c})$ also holds. Hence there exist, by Lemma 10.7, $f_{0}$ and $f_{1}$ indicating the beginning and the end for $f$.

Now let $A \Rightarrow \alpha, \alpha i \in \mathscr{A}$. This implies $\alpha i=e_{1}, A=e_{2},$. If $\alpha \neq e_{1}$ then (10.8.1) holds. Suppose (10:8.2a) holds, i. e. $e_{1} \rightarrow t, B \rightrightarrows e_{2},[\beta, \tau]$ is a structure of $[B, t]$. Then $B \Rightarrow \beta \equiv e_{2}$ which implies $\lambda \beta=1$, and (10.8.2b) holds. After suitable choice of $\omega$ such that $\omega[A, \alpha, i] \neq \alpha i$ as soon as $\alpha i=e_{1}$, we obtain by Theorem 10.8 that $\mathscr{A}$ is isolable. Since it is easy to see that every grammatical element $\left[e_{1}, t\right]$ has a unique structure, from Theorem 9.13 we obtain that $\mathscr{L}$ is structurally unambiguous if and only if $\mathscr{L}_{1}=\mathscr{L}_{\left\{e_{2}, e_{3}, \ldots, e_{n}\right\}}$ is such.

On putting $\bar{\Delta}_{i}=\Delta_{i-1}, \bar{n}=n-1, \bar{e}_{i}=e_{i-1}$ we see that $\mathscr{L}_{1}$ satisfies all the conditions originally imposed on $\mathscr{L}$. Hence $\mathscr{L}_{1}$ is structuraly unambiguous if and only if $\mathscr{L}_{2}=\mathscr{L}_{\left\{e_{3}, \ldots, e_{n}\right\}}$ is such. Since $\Lambda$ is a structurally unambiguous language, we obtain that $\mathscr{L}$ is structurally unambiguous.

\section{ISOLATING PARENTHESIZED SETS}

11.1. Definition. A subset $\mathscr{A}$ of $\mathbf{d} \mathscr{L}$ will be called parenthesized if $\mathscr{A}$ is complete, if for every $\alpha \in \bigcup \mathbf{r} \mathscr{L}, i \in \mathbf{d} \alpha, \alpha i \in \mathscr{A}$ either (10.8.1) or (10.8.2) holds, and if there are two disjoint subsets $L, R$ of $\mathbf{a}_{\mathbf{t}} \mathscr{L}$ such that $\alpha \in \bigcup \mathbf{r} \mathscr{L}$ and $\alpha i \in L(\in R)$ if and only if $\alpha \in \mathscr{L} A$, $A \in \mathscr{A}, i=1(=\lambda \alpha)$. 
11.2. Theorem. Every parenthesized subset of $\mathbf{d} \mathscr{L}$ is isolable.

Proof. Putting $Q=R$ we get from Lemma 10.4, the conditions of which follow from our assumptions, that $\mathscr{A}$ is $f$-recognizable with $f[A, t]=\{i ; i \in \mathbf{d} t, t i \in R\}$. Furthermore the assumptions of Lemma 10.7 are satisfied with $Q=R, k_{0} q=k_{1} q=$ $=0$ for every $q \in Q$, since for every $q$ in $Q$ condition (10.7.1) implies (10.7.2a) (because $\alpha^{(j+1, \lambda \alpha)}=\Lambda, u=\Lambda, \lambda u=k_{1} q$ ) and (10.7.3.b). Concerning the last condition, it follows from the fact that $f[A, u]=\Lambda$ implies $L \cap$ symb $\{u\}=\Lambda$ and that symb $_{\mathrm{b}} \mathscr{T}_{q} \subset L$ for $q \in Q$. Thus according to Lemma 10.7 there are $f_{0}$ and $f_{1}$ indicating the beginning and the end for $f$.

We see that the assumptions of Theorem 10.8 are satisfied if we choose suitably $\omega$ such that $\omega[A, \alpha, i] \neq \alpha i$ if $\alpha_{i} \in \mathscr{A}$ and $\omega[A, \alpha, i]=\alpha i$ otherwise.

11.3. Theorem. Let $\mathscr{A}$ be a parenthesizeds subset of $\mathbf{d} \mathscr{L}$, let $\mathscr{L}_{1}=\mathscr{L}_{\mathbf{d} \mathscr{L}-\mathscr{A}}$. For every $A \in \mathscr{A}, t \in \mathbf{t}(\mathscr{L}, A)$, let there be at most one structure $[\alpha, \tau]$ in $\mathscr{L}$ of $[A, t]$ satisfying either

$$
[\alpha, \tau]=[[A],[t]]
$$

or

2) $\quad[\alpha i, \tau i] \in \mathbf{g} \mathscr{L}_{1}$ for every $i \in \mathbf{d} \alpha$ such that $[\alpha i] \neq \tau i$.

Then the structural unambiguity of $\mathscr{L}_{1}$ is necessary and sufficient for that of $\mathscr{L}$.

Proof. The assertion follows from Theorem 9.13 since by Theorem $11.2, \mathscr{A}$ is isolable.

11.4. Example. Let $\Delta_{i}, e_{i}$ be as in Example 10.9, denote by $\mathscr{L}_{1}$ the language investigated (and denoted by $\mathscr{L}$ ) there. Let $p \in \Delta_{1}$, let $($, ) be two different elements not in a $\mathscr{L}_{1}$.

Let $\mathscr{L}$ be defined on the set $\{p\} \cup E$, let $\mathscr{L} p=\left\{\left[\left(e_{n}\right)\right]\right\}, \mathscr{L}_{E}=\mathscr{L}_{1}$. By an easy application of Theorem 11.3 we have that $\mathscr{L}$ is structurally unambiguous if $\mathscr{L}_{1}$ is such. According to Example $10.9, \mathscr{L}$ is structurally unambiguous.

\section{Bibliography}

[1] J. W. Backus, F. L. Bauer, J. Green, C. Katz, J. McCarthy, P. Naur (editor), A. J. Perlis. H. Rutishauser, K. Samelson, E. Vauquois, J. H. Wegstein, A. van Wijngaarden, M. Woodger: Report on the Algorithmic Language ALGOL 60. Numerische Mathematik 2 (1960), $106-136$.

[2] H. Bottenbruch: Übersetzung von algorithmischen Formelsprachen in die Programmsprachen von Rechenmaschinen. Zeit. für math. Logik und Grundlagen der Mathematik 4 (1958), 180-221.

[3] D. G. Cantor: On the Ambiguity Problem of Backus Systems. J. Assoc. Comp. Mach. 9 (1962), 477-479. 
[4] Noam Chomsky: On certain Formal Properties of Grammars. Information and Control 2 (1959), 137-167.

[5] Noam Chomsky: Three Models for the Description of Language. IRE Trans. 1, T-2, No. 3 (1956), 113-124. (Russian translation: Kibernetičeskij sbornik 2 (1961), 237-266, Izd. in. lit., Moskva.)

[6] N. Chomsky and M.P. Schützenberger: The Algebraic Theory of Context-free Languages. Computer Programming and Formal Systems (ed. by P. Braffort and D. Hirschberg), Amsterdam 1963.

[7] Martin Davis: Computability and Unsolvability. New York, McGraw-Hill 1958.

[8] Francois Genuys: Commentaires sur le langage Algol. Chiffres 5 (1962), 29-53.

[9] Sheita A. Greibach: The Undecidability of the Ambiguity Problem for Minimal Linear Grammars. Information and Control 6 (1963), 119-125.

[10] Peter Zilahy Ingerman: A Syntax-Oriented Compiler for Languages whose Syntax Is Expressible in Backus Normal Form, and Some Proposed Extensions Thereto. University of Pennsylvania, The Moore School of Electrical Engineering, Philadelphia, Pennsylvania, April 1963.

Резюме

О СТРУКТУРНОЙ ОДНОЗНАЧНОСТИ ФОРМАЛЬНЫХ ЯЗЫКОВ

ВАЦЛАВ ФАБИАН, (Václav Fabian), Прага

В работе изучается проблема (структурной) однозначности формальных языков несколько более общего класса, чем класс Хомского грамматик типа 2. Известно, что не существует алгоритм, который давал бы ответ на этот вопрос для каждого языка данного класса ([3], [6], [9]). Настоящая работа подходит к проблеме с другой стороны. В ней выводится ряд необходимых и достаточных условий для того, чтобы данный язык $\mathscr{L}$ был структурно однозначным. Многие из этих условий показывают, что $\mathscr{L}$ будет структурно однозначным тогда и только тогда, если другой, более простой язык обладает этим свойством. Подробно изучается также связь между структурной однозначностью языка и возможностью определения для этого языка семантики на основании рекуррентных соотношений, аналогичных тем, с помощью которых был определен синтаксис. 